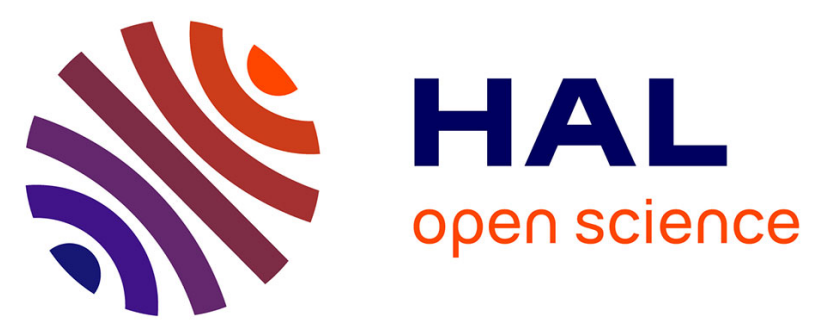

\title{
Dopamine Precursor Depletion in Healthy Volunteers Impairs Processing of Duration but Not Temporal Order
}

Morgane Chassignolle, Ljubica Jovanovic, Catherine Schmidt-Mutter, Guillaume Behr, Anne Giersch, Jennifer T Coull

\section{- To cite this version:}

Morgane Chassignolle, Ljubica Jovanovic, Catherine Schmidt-Mutter, Guillaume Behr, Anne Giersch, et al.. Dopamine Precursor Depletion in Healthy Volunteers Impairs Processing of Duration but Not Temporal Order. Journal of Cognitive Neuroscience, 2021, 33 (5), pp.946-963. 10.1162/jocn_a_01700. hal-03193831

\section{HAL Id: hal-03193831 \\ https://hal-amu.archives-ouvertes.fr/hal-03193831}

Submitted on 9 Apr 2021

HAL is a multi-disciplinary open access archive for the deposit and dissemination of scientific research documents, whether they are published or not. The documents may come from teaching and research institutions in France or abroad, or from public or private research centers.
L'archive ouverte pluridisciplinaire HAL, est destinée au dépôt et à la diffusion de documents scientifiques de niveau recherche, publiés ou non, émanant des établissements d'enseignement et de recherche français ou étrangers, des laboratoires publics ou privés.

$$
\text { Copyright }
$$




\title{
Dopamine Precursor Depletion in Healthy Volunteers Impairs Processing of Duration but Not Temporal Order
}

\author{
Morgane Chassignolle ${ }^{1}$, Ljubica Jovanovic ${ }^{2}$, Catherine Schmidt-Mutter ${ }^{3}$, \\ Guillaume Behr' ${ }^{2}$, Anne Giersch ${ }^{2}$, and Jennifer T. Coull ${ }^{1}$
}

\begin{abstract}
Studies in animals and humans have implicated the neurotransmitter dopamine in duration processing. However, very few studies have examined dopamine's involvement in other forms of temporal processing such as temporal order judgments. In a randomized within-subject placebo-controlled design, we used acute phenylalanine/tyrosine depletion (APTD) to reduce availability of the dopamine precursors tyrosine and phenylalanine in healthy human volunteers. As compared to a nutritionally balanced drink, APTD significantly impaired the ability to accurately reproduce interval duration in a temporal reproduction task. In addition, and confirming previous findings, the direction of error differed as a function of individual differences in underlying dopamine
\end{abstract}

\section{INTRODUCTION}

Processing temporal information allows us to estimate whether one event lasted longer than another (duration processing) or to determine which of two consecutive events occurred first (temporal order processing). In other words, time perception encompasses both the perception of duration and the perception of succession (Fraisse, 1984). These fundamental temporal processes allow us to interact with our dynamic environment by coordinating a unified perception of the way in which successive events unfold over time (Pöppel, 1997).

Impairments in processing the duration of events have been observed in a variety of neurological or psychiatric disorders, most notably Parkinson disease (Breska \& Ivry, 2018; Merchant, Luciana, Hooper, Majestic, \& Tuite, 2008; Harrington, Haaland, \& Hermanowitz, 1998; Artieda, Pastor, Lacruz, \& Obeso, 1992; Pastor, Artieda, Jahanshahi, \& Obeso, 1992; see Jones \& Jahanshahi, 2014, for a review), schizophrenia (Ciullo et al., 2018; Bolbecker et al., 2014; Roy, Grondin, \& Roy, 2012; Lee et al., 2009; Carroll, Boggs, O'Donnell, Shekhar, \& Hetrick, 2008; Elvevåg et al., 2003; see Thoenes \& Oberfeld, 2017, for a review), and

\footnotetext{
${ }^{1}$ Laboratoire des Neurosciences Cognitives (LNC), Aix-Marseille University \& CNRS, France, ${ }^{2}$ Inserm 1114, Strasbourg University, France, ${ }^{3} \mathrm{CIC}$ Inserm 1434, CHU Strasbourg, France
}

function. Specifically, APTD caused participants with low baseline dopamine precursor availability to overestimate the elapse of time, whereas those with high dopamine availability underestimated time. In contrast to these effects on duration processing, there were no significant effects of APTD on the accuracy of discriminating the temporal order of visual stimuli. This pattern of results does not simply represent an effect of APTD on motor, rather than perceptual, measures of timing because APTD had no effect on participants' ability to use temporal cues to speed RT. Our results demonstrate, for the first time in healthy volunteers, a dopaminergic dissociation in judging metrical (duration) versus ordinal (temporal order) aspects of time.

attention-deficit/hyperactivity disorder (ADHD; Suarez, Lopera, Pineda, \& Casini, 2013; Yang et al., 2007; Toplak, Rucklidge, Hetherington, John, \& Tannock, 2003; Smith, Taylor, Rogers, Newman, \& Rubia, 2002; see Noreika, Falter, \& Rubia, 2013, for a review). Intriguingly, all of these pathologies are linked to dysfunction of the dopaminergic system (Allman \& Meck, 2012). Moreover, motor and perceptual timing deficits in patients with Parkinson disease can be ameliorated with dopaminergic medication such as L-dopa (Torta et al., 2010; Merchant et al., 2008; Malapani, Deweer, \& Gibbon, 2002; Pastor et al., 1992), and studies in animals and healthy human volunteers confirm a clear link between dopamine function and timing ability (Agostino \& Cheng, 2016; Soares, Atallah, \& Paton, 2016; Coull, Cheng, \& Meck, 2011; Meck, 1996). For instance, rats overestimate duration after administration of dopaminergic agonists (e.g., the stimulant drug methamphetamine) but underestimate it after administration of antagonists (e.g., neuroleptic drugs like haloperidol; MacDonald \& Meck, 2005; Drew, Fairhurst, Malapani, Horvitz, \& Balsam, 2003; Meck, 1986, 1996; Maricq \& Church, 1983). In healthy human volunteers, dopaminergic antagonists impair temporal discrimination of auditory (Rammsayer, 1993, 1997, 1999) or visual (Coull, Hwang, Leyton, \& Dagher, 2012) stimuli, and perturb motor timing in temporal reproduction tasks (Coull, Hwang, Leyton, \& Dagher, 2013; Lake \& Meck, 2013). In addition, the 
effects of such exogenous dopaminergic manipulations on duration processing can vary as a function of endogenous dopamine levels. Coull, Hwang, et al. (2013) found that reducing the availability of the dopamine precursors tyrosine and phenylalanine slowed temporal reproduction times in participants with low baseline dopamine precursor levels but speeded them in participants with high baseline levels.

Although many studies demonstrate an important role for dopamine in duration processing, very few have investigated other facets of timing such as the processing of temporal order or simultaneity. Yet patients with schizophrenia have difficulty processing not only the duration but also the temporal order of events (Vatakis \& Bakou, 2015; Capa, Duval, Blaison, \& Giersch, 2014; Schwartz, Deutsch, Cohen, Warden, \& Deutsch, 1991). Moreover, Bellgrove et al. (2006) found that A2 allele TAq1 polymorphisms of the gene encoding dopamine beta hydroxylase (which catalyses the transformation of dopamine into noradrenaline) in ADHD is associated with deficits in temporal order judgments (TOJs). Finally, temporal discrimination thresholds (i.e., the minimum time needed to perceive two stimuli as temporally separate) in simultaneity judgment tasks are improved with dopamine medication (i.e., L-dopa) in patients with Parkinson disease (Lee, Kim, \& Lyoo, 2005; Artieda et al., 1992).

These patient studies therefore indicate that the dopaminergic system might be involved not only in perceiving the duration of events but also in perceiving the succession of events, that is, how events are located in time relative to one another. However, it is still unclear whether the dopaminergic system plays a role in the perception of succession because very few psychopharmacological studies have been conducted. In one study of duration processing in healthy volunteers, Rammsayer (2009) used an auditory simultaneity task as a control and found that, although pergolide (dopamine agonist) altered the perception of duration, it had no effect on auditory fusion thresholds (i.e., the interval separating two consecutive stimuli that results in the perception of a single sound). Similarly, White et al. (2014) found no effect of the D2/D3 antagonist amisulpride on auditory simultaneity processing in healthy volunteers, although impairments were found in patients with schizophrenia. It is, of course, possible that perturbations in temporal thresholds in patients are related to pathological factors other than dopaminergic function and that the dopaminergic system is simply not involved in the perception of succession. We therefore tested, for the first time, the effects of dopaminergic manipulation on TOJs in healthy volunteers.

We modulated dopaminergic function in a randomized within-subject, double-blind, placebo-controlled design using acute phenylalanine/tyrosine depletion (APTD). APTD is an amino acid drink that lowers levels of the dopamine precursors phenylalanine and tyrosine, and reduces striatal dopamine levels in animals (McTavish, Cowen, \& Sharp, 1999) and humans (Leyton et al., 2004;
Montgomery, McTavish, Cowen, \& Grasby, 2003). By measuring the effects of APTD on temporal reproduction and TOJ within the same experiment, we were able to compare the effects of dopaminergic manipulation on the processing of duration versus the processing of succession. To complement prior studies that explored succession processing in the auditory domain (White et al., 2014; Rammsayer, 2009), we measured effects of APTD on temporal order processing in the visual domain. In our TOJ task, participants had to judge which of two consecutive stimuli (separated by 17-83 $\mathrm{msec}$ ) had appeared first. To control for nontemporal cognitive processes, participants also performed a spatial position judgment (SPJ) task in which participants had to judge which of two stimuli appeared slightly more to the left of the other (similar to Vernier acuity; Levi, Klein, \& Aitsebaomo, 1985; Westheimer \& McKee, 1977). Therefore, both TOJ and SPJ tasks required a discrimination judgment about the relative location of two stimuli, but this discrimination was made in terms of either location in time (TOJ) or location in space (SPJ). We reasoned that if dopamine were involved in temporal order processing, APTD would impair performance on the TOJ task but would not necessarily affect performance on the SPJ task.

In the temporal reproduction task, participants first learned a "standard" interval of time between two visual stimuli (600 or $1400 \mathrm{msec}$ ), and then later reproduced this interval by making a timed motor response. Importantly, the standard interval was learned at the beginning of the experimental session before dopaminergic manipulation. This contrasts with the "roving standard" design (Wearden \& Bray, 2001) used in the previous APTD study of temporal reproduction (Coull, Hwang, et al., 2013), in which the interval was both learned and reproduced within a single trial after dopaminergic manipulation. As such, results could have reflected the effect of APTD either on the representation of duration that had been encoded into memory and/or the retrieval and reproduction of that duration from memory. The design of the current study allowed us to dissociate these two processing stages (Malapani et al., 2002): Because decreases in dopamine precursor availability occurred after the standard interval had been learned, any effects of APTD on performance must necessarily reflect effects on retrieval and reproduction rather than encoding. This approach allowed us to confirm whether APTD affected the encoding of duration only (Coull, Hwang, et al., 2013; Coull et al., 2012) or whether it could also affect its retrieval and reproduction.

\section{METHODS}

\section{Participants}

Twenty-six healthy participants (mean age $=25.54$ years; $S D=5.30$; nine men) volunteered for this study. They signed written, informed consent forms, accepting to participate in the study, which had been approved by a 
Figure 1. Timeline for one experimental session. The day before the session, participants followed a low-protein diet then fasted from midnight. Upon arrival in the laboratory, the procedure was explained and a baseline blood sample was drawn. Participants then consumed an amino acid drink that was either nutritionally balanced (BAL) or deficient in tyrosine and phenylalanine (APTD). After a 4-hr wait, a second blood sample was taken just prior to test start. Cognitive testing then lasted approximately 1 hr. VAS ratings were acquired at baseline and at the end of the cognitive test session. All participants performed two experimental sessions, with treatment order counterbalanced across participants.

national ethics committee (Comité de Protection des Personnes Nord-Ouest I, University of Rouen, protocol \# CPP 0013/2017-2016-A01598-43). They had normal or corrected-to-normal vision and were not color-blind, and female participants performed a pregnancy test before each test session to verify that they were not pregnant. Before being included, a psychiatrist ensured that participants did not suffer from neurological or psychiatric disorders and scores on the Beck Depression Inventory and Community Assessment of Psychic Experiences scale confirmed they had no depressive or psychotic symptoms. Six participants had incomplete data sets because of vomiting during one of the test sessions and so were excluded from all analyses. This side-effect has already been reported in previous studies (Leyton, 2010; Leyton et al., 2000). One further participant fainted during the initial blood test and so was excluded from the study. The final sample therefore comprised 19 participants (mean age $=26.26 ; S D=5.79$; eight men). This sample size is similar to previously published dopaminergic studies of timing in healthy volunteers (Tomassini, Ruge, Galea, Penny, \& Bestmann, 2016; Coull, Hwang, et al., 2013; Lake \& Meck, 2013; Coull et al., 2012).

\section{APTD Manipulation and Experimental Design}

Testing took place in the Clinical Investigation Centre of Strasbourg University Hospital. Each participant performed two test sessions separated by a minimum of 1 week (mean intersession interval $=10.26$ days; range: 7-22 days). After a randomized, within-subject, doubleblind, placebo-controlled crossover design, participants ingested an APTD drink in one session and a nutritionally balanced (BAL) drink in the other. The aim of the APTD manipulation is to induce a significant decrease in dopamine precursor levels and, hence, dopamine availability (Leyton et al., 2004; Montgomery et al., 2003; Harmer, McTavish, Clark, Goodwin, \& Cowen, 2001; McTavish et al., 1999) in the APTD session compared to the BAL session.

The BAL drink consisted of a diluted mixture of eight amino acids: isoleucine (15 g), leucine (22.5 g), lysine (17.5 g), phenylalanine (12.5 g), threonine (10 g), tryptophan (2.5 g), tyrosine (12.5 g), and valine (17.5 g), all dissolved in $400 \mathrm{~mL}$ of water. The APTD drink was composed of the same amino acids except Phenylalanine and Tyrosine, which are the two precursors of dopamine. Methionine ( $5 \mathrm{~g}$ ) was given (in capsule form because of its unpleasant taste and smell) just prior to drink ingestion. Because women generally have lower body weight than men, doses of amino acids were lowered by $15 \%$ for female participants.

Both sessions (APTD and BAL) were conducted in the same way (Figure 1) except that, in one session, participants had the APTD drink and, in the other, the BAL drink, with treatment order being counterbalanced across participants. To maximize the effects of the amino acid manipulation, participants followed a low-protein diet (primarily fruit, vegetables, and bread) the day before the test session and fasted (other than water) from midnight. They were asked to abstain from alcohol the day before the test session and to have a maximum of three caffeinated drinks. All participants were tested at roughly the same time of day (baseline measurements in the morning and the cognitive test phase in the early afternoon). When participants arrived at the testing center, a blood test was taken prior to ingestion of the drink and capsules, in order to quantify baseline plasma amino acid levels. Visual Analogue Scales (VASs) of arousal and emotion were also measured at this time. After ingestion of the capsules and drink, participants waited quietly for $4 \mathrm{hr}$ to allow sufficient time for the drink to take effect. A second blood test was then taken to quantify plasma amino acid levels immediately prior to the cognitive test phase. Cognitive task performance therefore began a little more than $4 \mathrm{hr}$ after the first sip of the drink (average: $4 \mathrm{hr} 09 \mathrm{~min}$ ), and cognitive testing lasted approximately $1 \mathrm{hr}$. Finally, a second set of VAS measurements was taken right at the end of the test session.

All blood samples were centrifuged immediately $\left(24^{\circ} \mathrm{C}\right.$, $3000 \mathrm{rpm}, 10 \mathrm{~min}$ ), and plasma was stored at $-80^{\circ} \mathrm{C}$ until analysis. Plasma concentrations of phenylalanine, tyrosine, and other large neutral amino acids (LNAAs: leucine, isoleucine, methionine, valine, and tryptophan) were measured by liquid chromatography-mass spectrometry (Waters Xevo TQ-S micro). 


\section{Experimental Tasks}

The tasks were programmed with E-Prime 2.0 and 3.0 software. Visual stimuli were presented on a CRT screen with $800 \times 600$ spatial resolution and $60-\mathrm{Hz}$ temporal resolution. All visual stimuli were presented on a black background.

Participants performed three timing tasks: temporal reproduction, temporal orienting, and TOJ. Presentation order of tasks was randomized across participants, with the proviso that the temporal reproduction task was always performed before the temporal orienting task (to prevent participants from using the intervals presented in the orienting task to guide their responses in the reproduction task). Participants were trained on (reminded of) all tasks at the beginning of the first (and second) experimental sessions.

\section{Temporal Reproduction Task}

In the temporal reproduction task, participants had to reproduce two intervals of time (600 or $1400 \mathrm{msec}$ ) that had been learned at the beginning of the experimental session before dopamine precursor availability had been manipulated. Because the dopaminergic manipulation could not therefore affect the initial encoding of the interval, any effects of APTD on performance would have to reflect effects on its retrieval and reproduction. During the initial learning phase, a specific visual cue was associated with either the 600- or 1400-msec interval. At the beginning of each trial, a background image, comprising a central cueing stimulus (two concentric circles) and two peripheral squares, was presented for 1000-2000 msec (Figure 2A). One of the two concentric circles then brightened for $200 \mathrm{msec}$. If the smaller interior circle brightened, the target to which participants had to respond appeared after a short interval of $600 \mathrm{msec}$. If, on the other hand, the larger outer circle brightened, the target appeared after a longer 1400-msec interval. Participants had to respond as quickly as possible to the appearance of the target (a "+" presented inside both squares) by pressing the left mouse button. The 600- and 1400-msec intervals were learned in separate blocks. Each of these blocks consisted of 10 learning trials, as described above, followed by 10 reproduction trials in which the cue appeared and participants then had to press the mouse button when they believed the learned interval had elapsed. As soon as the participant pressed the button, the target appeared to provide visual feedback that their response had been registered. After these two learning blocks, participants were familiarized with the task by performing 20 reproduction trials in which the 600- and 1400 -msec trials were presented in random order (10 trials each). During the test phase $4 \mathrm{hr}$ later, participants performed 60 reproduction trials (30 trials each of the 600and 1400-msec trials, presented in random order).

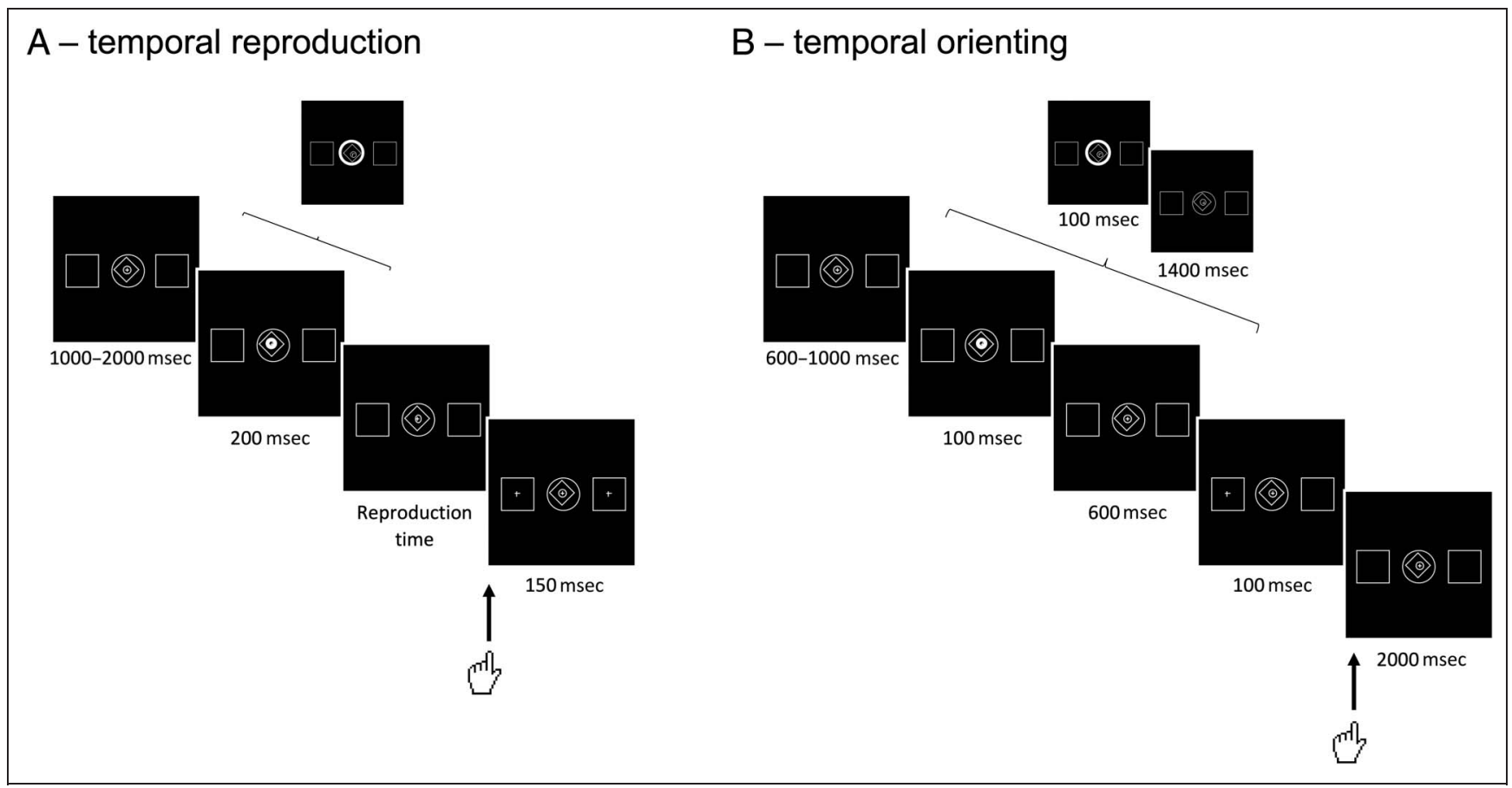

Figure 2. (A) Temporal reproduction task: Participants reproduced a short (600 msec) or long $(1400 \mathrm{msec})$ interval that had previously been associated with a visual cue at the beginning of the experimental session. Upon presentation of the cue (either a small or large [inset] central circle), participants initiated a button-press response when they estimated that the cued interval had elapsed. Two peripheral crosses immediately appeared simply to confirm that their response had been registered. (B) Temporal orienting task: Participants used the small or large (inset) central cue to predict whether the target would appear after a short $(600 \mathrm{msec})$ or long $(1400 \mathrm{msec})$ interval. Upon presentation of the target in either the left or right box, participants made a speeded button-press response. In $20 \%$ of trials, the target appeared at the time that was not predicted by the cue ("invalid" trials). 


\section{Temporal Orienting Task}

To investigate whether APTD affected other forms of motor timing, we compared the effects of APTD on temporal reproduction to its effects on temporal orienting (Coull \& Nobre, 1998). In both tasks, participants had to access representations of short (600 msec) or long (1400 msec) intervals, each of which had been associated with distinct visual cues. In the reproduction task, participants had to initiate a motor response after the delay corresponding to the cue. By contrast, in the orienting task, participants had to react as quickly as possible to an imperative target that appeared after the delay predicted by the cue. These two different tasks of motor timing are characterized respectively by self-initiated versus stimulus-guided motor responding (Coull, Davranche, Nazarian, \& Vidal, 2013). Stimuli in the temporal orienting task were very similar to those used in the temporal reproduction task (Figure 2B). The only difference was that the target (a single "+") appeared in either the left or right box. In the learning phase, the associations between small and large circles, and 600- and 1400-msec intervals, respectively, were learned in separate blocks of 24 trials. Participants were then familiarized with the orienting task by performing 24 trials in which the 600- and 1400-msec trials were presented in random order (12 trials each). In these trials, participants were instructed to use the brief $(100 \mathrm{msec})$ presentation of the small or large cue to predict whether the target would be presented after a short or long interval, respectively, so as to respond as quickly as possible to the target. During the test phase $4 \mathrm{hr}$ later, participants performed 120 orienting trials. In $96(80 \%)$ of these trials, the cue correctly predicted target onset time ("valid trials"), whereas in the remaining 24 trials (20\%), the cue incorrectly predicted target onset time ("invalid trials"). In both valid and invalid trials, the target appeared after $600 \mathrm{msec}$ for half of the trials and after $1400 \mathrm{msec}$ for the other half. Target position (left/ right) was counterbalanced across all four trial types (i.e., across the factorial combination of interval duration and cue validity). Trials were presented in random order. During the learning phase, cues were 100\% valid.

To control for nontemporal sensorimotor processes, participants also performed a spatial version of this task in which the cue predicts where, rather than when, the target will appear. This control condition was equivalent to the Posner spatial orienting of attention task (Posner, Snyder, \& Davidson, 1980). In this spatial condition, the visual cue was a brief $(100 \mathrm{msec})$ brightening of the left or right side of the central diamond, which formed a leftward or rightward facing arrow. Participants were instructed to use the presentation of the left- or right-sided cue to predict whether the target ("+") would appear in the left- or right-hand box, in order to make a speeded response to the target's appearance. During the test phase, participants performed 120 spatial trials (96:24 valid:invalid trials; 50:50 left:right target). Target onset time (600/1400 msec) was counterbalanced across all four trial types (i.e., across the factorial combination of target position and cue validity). Trials were presented in random order. During the learning phase, cues were $100 \%$ valid.

\section{TOJ Task}

In the TOJ task, participants had to judge which of two consecutive stimuli appeared first (Figure 3A). In all trials, a background image comprising two squares, one above and one below a central cross, was presented on the screen. At the beginning of each trial, each square was colored red or green in a particular order: red then green, or green then red, with color being independent of position (e.g., red could first appear in either the top or the bottom square followed by green in the remaining square). The SOA between presentation of the first and second colors was $17,33,50,66$, or $83 \mathrm{msec}$. SOAs varied in a randomized manner across trials. The temporal precision of stimulus presentation was verified by a photodiode prior to the experiment. The first color remained on the screen throughout the SOA. Upon onset of the second color, both colors remained together on the screen for $250 \mathrm{msec}$. The screen then reverted to the background image for $133 \mathrm{msec}$, before the response screen appeared for a maximum of $3 \mathrm{sec}$. The response screen comprised the words "VERT" and "ROUGE" ("green" and "red" in French), which appeared simultaneously on the left and right of the central cross. The relative position of the words was randomized across trials (e.g., "VERT" could appear on either the left or right from one trial to the next, with "ROUGE" appearing on the opposite side). When the response screen appeared, participants had to press the left or right arrow of the computer keyboard, with the index finger of their left or right hand, respectively, according to the position of the word describing the color that had been presented first (e.g., if the first color was green and the word "VERT" appeared on the right, participants had to press the right arrow button). Upon registration of the response, the response screen disappeared and the background image was presented for a variable intertrial interval (800-1200 msec). We recorded both accuracy and RT of the response. In total, 160 trials comprising 32 trials of each of the five SOAs (16 "green first" and 16 "red first" trials per SOA) were presented in random order. To familiarize participants with the task, they first performed 40 trials (20 green-first and 20 red-first trials), comprising a randomized presentation of eight trials per SOA.

To control for nontemporal cognitive processes, such as sensorimotor processing or decision making, participants also performed a spatial version of this task. In the SPJ condition, participants had to judge which of two stimuli appeared more to the left of the other (Figure 3B). At the beginning of each trial, a red and a green colored square appeared simultaneously for $66 \mathrm{msec}$, positioned diagonally above and below a central cross. One colored square appeared slightly to the left and the other slightly to the right of the central cross $\left(0.29^{\circ}\right.$ visual angle). Both the 


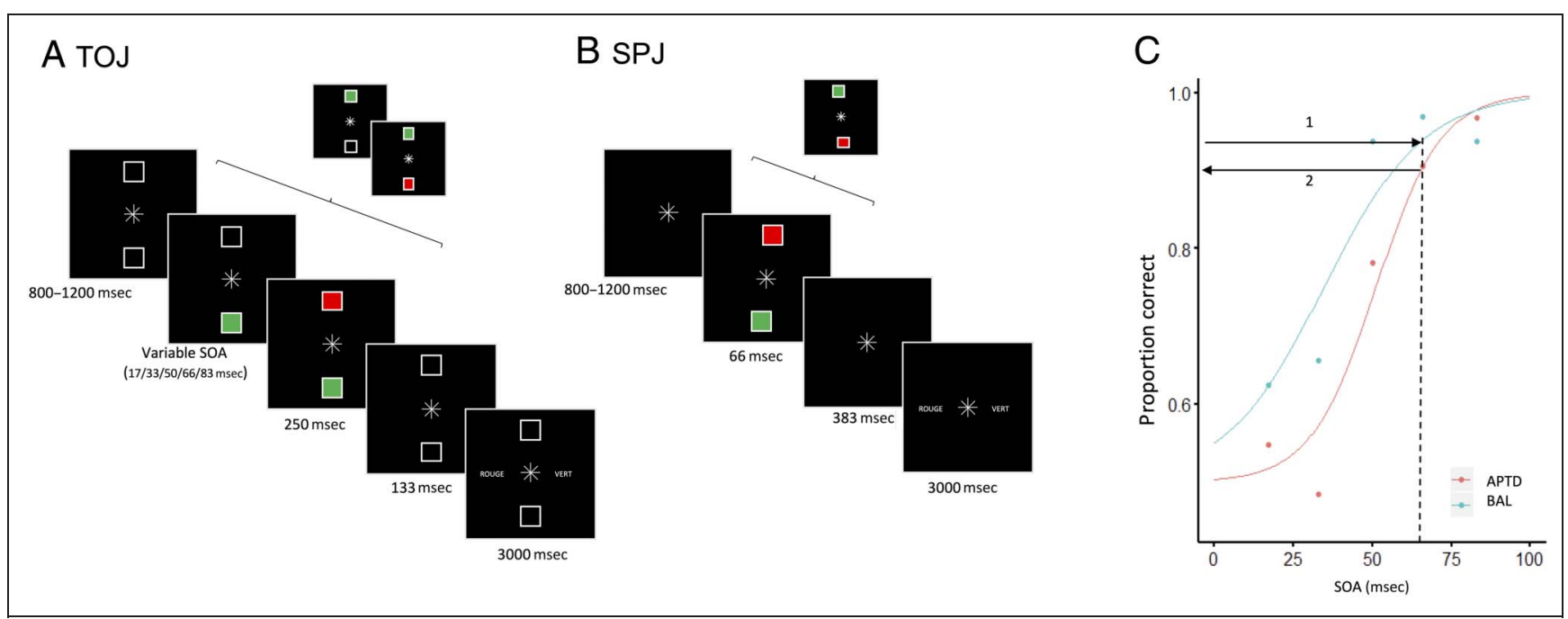

Figure 3. (A) TOJ task: Two colored squares appeared consecutively, separated by a variable SOA. The order of presentation was either green first or red first. The response screen comprised the words red and green ("rouge" and "vert" in French) located on the left and right of the screen, and participants responded with the left or right hand according to the position of the word describing the color they had perceived first. In this example, the green square appears first and so participants should respond with their right hand (corresponding to the right-hand location of the word "vert"). Color was independent of position (see inset). (B) SPJ task: Two colored squares appeared simultaneously, with either the green or red square being located slightly more to the left of center. The leftmost square could appear either above or below the central cross (see inset). Participants had to respond with the left or right hand according to the position of the word describing the color they perceived to have been presented more to the left. In this example, the green square appears on the left and so participants should respond with the right hand ("vert"). (C) Data points indicate the proportion of correct TOJ responses at each of the SOAs in the APTD and BAL sessions for one representative participant, and curves represent the psychometric fit of these points. Black arrows represent the values used to match performance of the TOJ and SPJ tasks on a participant-by-

participant basis. The first black arrow corresponds to the proportion of correct responses in the SPJ task during the BAL session and identified the SOA that would be needed to achieve the same level of performance in the TOJ task during the BAL session (vertical dashed line). The second black arrow corresponds to the level of TOJ performance that would be found at this SOA in the APTD session.

vertical (top/bottom) and horizontal (left/right) position of the colored squares was randomized (e.g., the red or green square could appear at the top left, top right, bottom left or bottom right across trials). Because the SPJ condition was included simply to a control for nontemporal processes, the spatial eccentricity of red and green stimuli was not varied across trials. After the colored squares had disappeared, the central cross was presented alone for an interval of $383 \mathrm{msec}$, and the response screen then appeared for a maximum of $3 \mathrm{sec}$. The response screen comprised the words "VERT" and "ROUGE," as in the TOJ condition, and participants had to press one of the two arrow keys with their left or right index fingers according to the position of the word describing the color of the leftmost square (e.g., if the leftmost color was green and the word "VERT" appeared to the right of the central cross, participants had to press the right arrow button). Upon registration of the response, the response screen disappeared and the central cross was presented for a variable intertrial interval (800-1200 msec). We recorded both accuracy and RT of the response. In total, 32 trials (16 "red left" and 16 "green left" trials) were presented in random order. Participants were familiarized with this condition by performing 16 "red left" and 16 "green left" trials.

\section{Visual Analogue Scales (VAS)}

Participants completed VASs (Bond \& Lader, 1974) to quantify subjective levels of arousal and anxiety before (baseline) and after (test phase) ingestion of the drink. Participants were asked to mark their subjective state on one of three $10-\mathrm{cm}$ scales, bounded by two adjectives (alert-drowsy, calm-excited, and contented-discontented). Measurements were taken immediately after the first blood test and after performing the cognitive tasks.

\section{Data Analysis}

\section{Dopamine Precursor Availability}

In rodents, the effects of APTD on dopamine precursor synthesis in the brain can be measured directly (McTavish et al., 1999). In humans, the fact that amino acids compete for transport across the blood-brain barrier (Harmer et al., 2001; Oldendorf \& Szabo, 1976) means that brain availability of dopamine precursors can be approximated by the relative plasma concentration of tyrosine to that of other LNAAs. Indeed, the magnitude of APTD-induced decreases in the ratio of tyrosine to LNAAs correlates with increases in $\left[{ }^{11} \mathrm{C}\right]$ raclopride binding in humans (Montgomery et al., 2003), providing a strong link between measures of dopamine precursor availability in the blood and dopamine function in the brain. Therefore, for each participant and each experimental session, we calculated dopamine precursor availability by dividing the plasma concentration of tyrosine by the sum $(\Sigma)$ of the plasma concentrations of all other LNAAs (phenylalanine, leucine, isoleucine, methionine, valine, and tryptophan). Plasma concentrations of tyrosine 
and phenylalanine, as well as the tyr: 2 LNAA ratio, were measured at baseline and just before the cognitive test session ( $4 \mathrm{hr}$ after the ingestion of the drink) and analyzed in SPSS (IBM) using repeated-measures ANOVAs, with Drink (BAL/APTD) and Time (baseline/test) as within-subject factors.

\section{Experimental Tasks}

Temporal Reproduction Task. We first excluded all trials with response times $<100 \mathrm{msec}$. For each participant and each experimental session, we calculated the mean and standard deviation (SD) of reproduction times for the short (600 msec) and long (1400 msec) intervals. From these means, we also calculated the absolute (unsigned) error in performance as the difference between the mean reproduction time and the corresponding standard interval (600 or $1400 \mathrm{msec}$ ). We used SPSS to conduct repeated-measures ANOVAs on means, SDs, and absolute errors with Drink (APTD/BAL) and Interval Duration (600/ $1400 \mathrm{msec}$ ) as within-subject factors.

Previously, APTD has been shown to differentially modulate temporal reproduction depending upon individual participants' baseline level of dopamine precursor availability (Coull, Hwang, et al., 2013). In order to confirm this finding in the current study, we used the same methodology as Coull, Hwang, et al. (2013) and divided participants into low or high dopamine precursor availability groups using a median split of the tyr: $\Sigma$ LNAA data. As in the previous study, we defined individual levels of baseline dopamine precursor availability by averaging the tyr: $\Sigma$ LNAA ratio over both sessions for each participant (Figure 4A). We then conducted a repeated-measures ANOVA on mean reproduction times with Drink (APTD/ BAL) and Interval Duration (600/1400 msec) as withinsubject factors and baseline Dopamine Precursor Availability (low/high) as a between-subjects factor.

Temporal Orienting Task. For each participant and each experimental session, we calculated the mean RT for validly or invalidly cued targets that appeared after short (600 msec) or long (1400 msec) intervals in the temporal orienting task. Mean RTs were also calculated for validly or invalidly cued targets that appeared in either the left- or right-hand box in the spatial orienting task. We excluded all trials with response times $<100$ msec. We then used SPSS to analyze mean RTs from temporal and spatial orienting tasks separately, using repeated-measures ANOVAs with Drink (APTD/BAL), Validity (valid/invalid), and Interval Duration (600/1400 msec) as within-subject factors for the temporal orienting task, or Drink, Validity, and Target side (left/right) as within-subject factors for the spatial orienting task. Dopamine Precursor Availability (low/high) was included as a between-subjects factor in both ANOVAs.

TOJ Task. We measured the proportion of correct responses at each SOA $(17,33,50,66$, and $83 \mathrm{msec})$ for each participant and each experimental session. These data were initially analyzed using a $2 \times 5 \times 2$ repeated-measures ANOVA in SPSS with Drink (APTD/BAL) and SOA as within-subject factors and Dopamine Precursor Availability (low/high) as a between-subjects factor. We then fitted

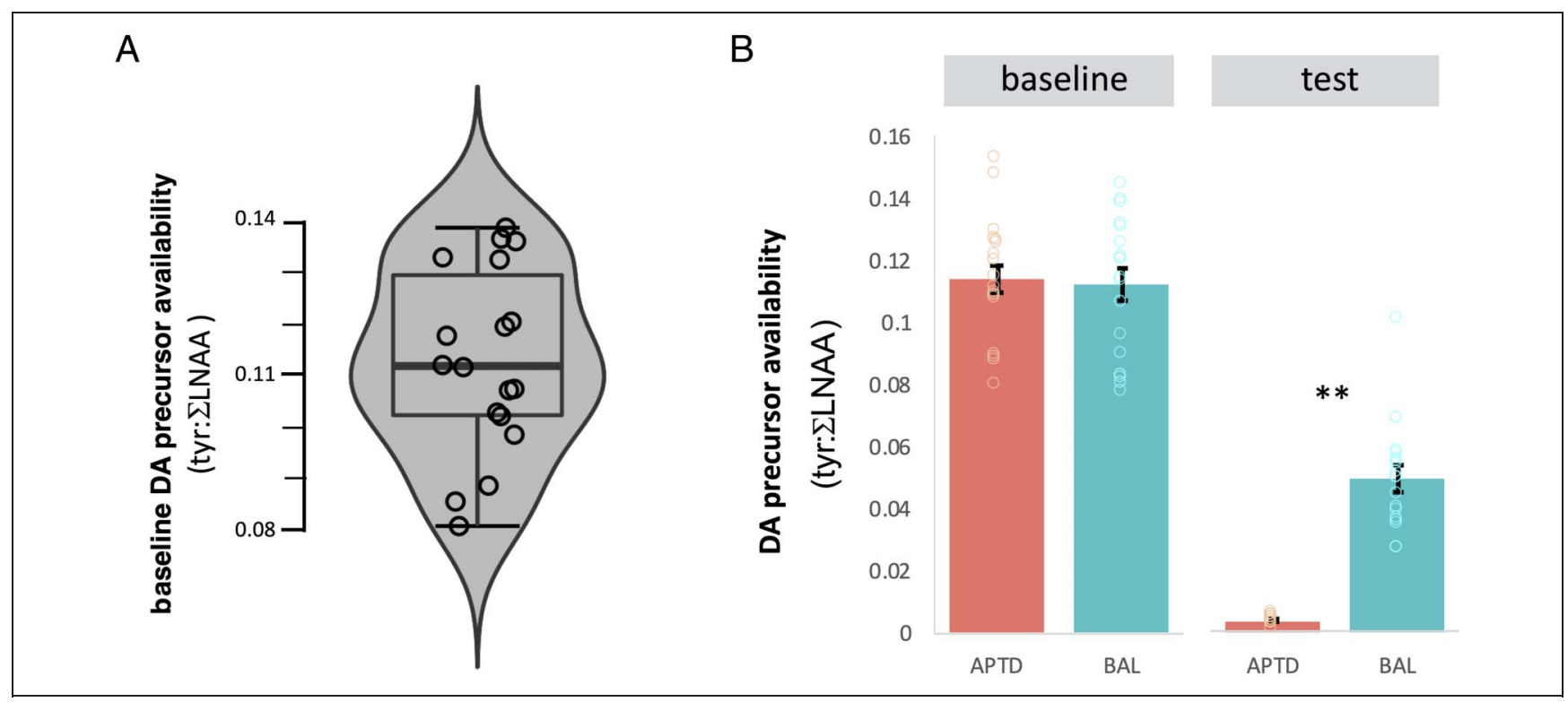

Figure 4. (A) Distribution of baseline dopamine precursor availability (tyrosine plasma concentration divided by the sum of plasma concentrations of all other LNAAs, averaged over both experimental sessions). Circles represent the averaged baseline dopamine precursor availability for each individual participant. The black horizontal line indicates the median, and whiskers delimit 1.5 times the interquartile range. The violin plot was constructed using JASP software (Version 0.14). (B) Mean ratio of tyrosine to all other amino acids (tyr: $L \mathrm{LNAA}$ ) at baseline and just before cognitive testing ( $4 \mathrm{hr}$ after ingestion of the drink) during the APTD and BAL sessions. There was no difference in brain availability of dopamine precursors between the APTD and BAL sessions at baseline, but there was a significant difference (**) just before test start. Columns reflect group means, and open circles indicate the level of dopamine precursor availability for individual participants. Error bars represent the SEM. 
psychometric curves to data from each individual participant in each experimental session (APTD/BAL) using the logistic function (Equation 1) from the quickpsy package in R. To verify the goodness of each fit, we calculated the coefficient of determination $\left(R^{2}\right)$ via the Method 1 of Kvålseth (1985). From each curve fit, we obtained both a temporal order threshold ( $\alpha$ parameter), corresponding to the SOA at which the participant would have responded correctly 75\% of the time (Ulrich \& Miller, 2004; Kanabus, Szelag, Rojek, \& Pöppel, 2002), and the slope of the distribution ( $\beta$ parameter), which reflects temporal sensitivity. Using SPSS, we then conducted $2 \times 2$ repeated-measures ANOVAs on these two parameters with Drink (APTD/BAL) as a within-subject factor and Dopamine Precursor Availability (low/high) as a between-subjects factor.

$$
y=\gamma+(1-\gamma) \cdot\left(\frac{1}{1+\exp (-\beta \cdot(x-\alpha))}\right)
$$

In the logistic function used to fit TOJ data, $\alpha, \beta$, and $\gamma$ parameters correspond respectively to the threshold, the slope, and the guess rate. Guess rate was fixed at 0.5.

We compared the effects of APTD on TOJ and SPJ tasks directly, while controlling for task difficulty, by using the psychometric curve of TOJ performance in the BAL session to calculate the SOA that would correspond to the same level of performance (proportion correct) as that observed in the BAL session of the SPJ task. This procedure was conducted for each individual participant (Figure 3C). We then used this participant-specific SOA and the psychometric curves of the APTD session to interpolate what this participant's TOJ performance (proportion correct) would be at this particular SOA in the APTD session. In this way, we ensured that the effects of APTD were being compared on two tasks matched for difficulty on a participantby-participant basis. To measure the effects of APTD on task performance, we conducted a $2 \times 2$ repeated-measures ANOVA in SPSS on the proportion of correct responses with Drink (APTD or BAL) and Task (TOJ, SPJ) as withinsubject factors.

VAS. For each of the three VASs, we calculated the distance of the participant's mark from the left-hand side of the 10 - $\mathrm{cm}$ scale and analyzed these data using $2 \times 2 \times 2$ ANOVAs with Drink (APTD/BAL) and Time (baseline/test) as within-subject factors and Dopamine Precursor Availability (low/high) as a between-subjects factor.

\section{RESULTS}

One of the participants was identified as an outlier for performance of all cognitive tasks, and so her data were excluded from all analyses. The remaining 18 participants were divided into low or high baseline dopamine precursor availability groups using a median split of the tyr: $\Sigma$ LNAA ratio, averaged over BAL and APTD sessions (Figure 4A). Levels of baseline dopamine precursor availability in the BAL session correlated with those in the APTD session across participants (Spearman $R=.50 ; p=.018$ ), confirming that individual differences in the tyr: $\Sigma$ LNAA ratio were stable from one session to the next. ${ }^{1}$

\section{Dopamine Precursor Concentrations}

Measurement of tyrosine and phenylalanine plasma concentration revealed a main effect of the Drink $(F(1,17)=99.58$; $p<.001$; partial eta $=.85$ for tyrosine and $F(1,17)=156.26$; $p<.001$; partial eta $=.90$ for phenylalanine), a main effect of Time $(F(1,17)=18.50 ; p<.001$; partial eta $=.52$ for tyrosine and $F(1,17)=61.98 ; p<.001$; partial eta $=.79$ for phenylalanine), and an interaction between Drink and Time $(F(1,17)=136.71 ; p<.001 ;$ partial eta $=.89$ for tyrosine and $F(1,17)=146.26 ; p<.001$; partial eta $=.90$ for phenylalanine). These results indicated that, in the BAL session, plasma concentrations increased significantly $(p<.001)$ from baseline to test for both tyrosine $(67.73 \mu \mathrm{mol} / \mathrm{L}$ vs. $169.76 \mu \mathrm{mol} / \mathrm{L})$ and phenylalanine $(69.16 \mu \mathrm{mol} / \mathrm{L}$ vs. $322.54 \mu \mathrm{mol} / \mathrm{L}$ ) but conversely, in the APTD session, concentrations of both tyrosine $(61.96 \mu \mathrm{mol} / \mathrm{L}$ vs. $15.27 \mu \mathrm{mol} / \mathrm{L})$ and phenylalanine $(63.37 \mu \mathrm{mol} / \mathrm{L}$ vs. $7.33 \mu \mathrm{mol} / \mathrm{L})$ decreased significantly $(p<.001)$.

More importantly, brain availability of dopamine precursors was indexed by the ratio of tyrosine plasma concentration to the sum of all other amino acids (tyr: $\Sigma$ LNAA). Analysis of this ratio revealed a main effect of Drink, $F(1$, $17)=34.35 ; p<.001 ;$ partial eta $=.67$; a main effect of Time, $F(1,17)=489.39 ; p<.001$; partial eta $=.97$; and an interaction between Drink and Time, $F(1,17)=87.15$; $p<.001$; partial eta $=.84$. Although there was no difference between BAL and APTD at baseline $(p=.72)$, there was a significant difference at test $(p<.001$; Figure $4 \mathrm{~B})$.

\section{Temporal Reproduction}

Three participants were identified as outliers (green dots in Figure 5A), with unusually long mean reproduction times for the 1400-msec condition of the BAL session. They were therefore excluded from further statistical analyses of temporal reproduction data.

The repeated-measures ANOVA of mean reproduction times revealed, as expected, a main effect of Interval Duration, $F(1,13)=205.75 ; p<.001$; partial eta $=.94$, indicating that reproduction times were shorter in the 600-msec condition compared to the 1400-msec condition. However, there was no significant main effect of the Drink, $F(1,13)=0.35 ; p=.55$; partial eta $=.02$, nor interaction between Drink and Interval Duration, $F(1,13)=1.35$; $p=$ .27 ; partial eta $=.09$.

The repeated-measures ANOVA of $S D$ revealed the expected effect of Interval Duration, $F(1,13)=37.05$; $p<.001$; partial eta $=.73$. In accordance with the scalar property of timing, variability in temporal reproduction time was greater for reproduction of 1400 -msec intervals $(M=248.05 \mathrm{msec}, S E=18.67)$ than 600-msec intervals $(M=167.8 \mathrm{msec}, S E=14.29)$. Importantly, there was no main effect of Drink on the variability of reproduction 


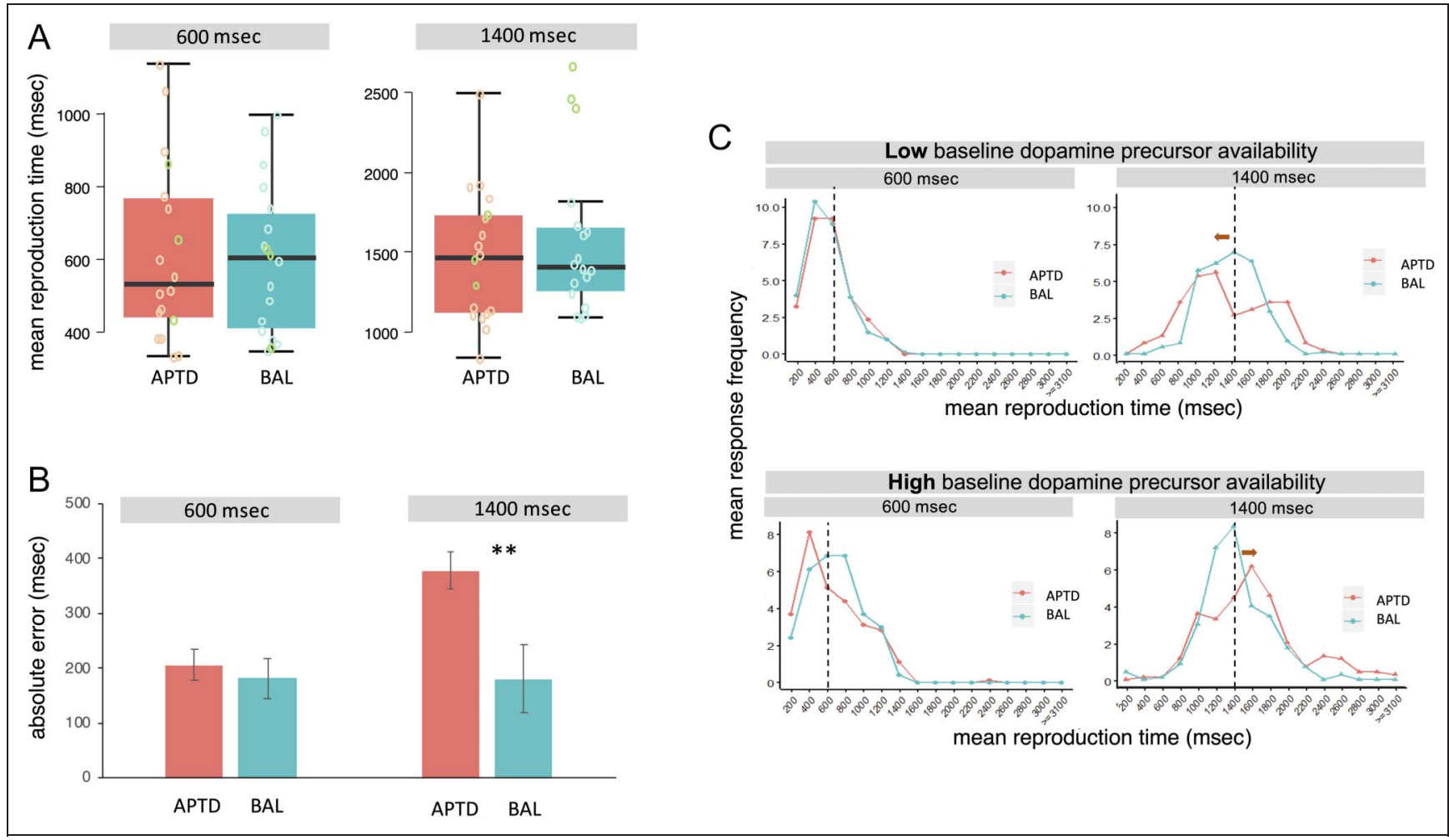

Figure 5. Temporal reproduction task. (A) Distribution of mean reproduction times of 600- or 1400-msec intervals across the entire group of 18 participants in either the APTD or BAL sessions. Each circle represents the mean reproduction time for 1 of the 18 participants. Three of the participants (green circles) produced exceedingly long reproduction times for the 1400-msec interval in the BAL session (furthest right plot) and were excluded from statistical analyses. Black horizontal lines indicate the median and whiskers delimit 1.5 times the interquartile range. Boxplots were constructed with JASP software (Version 0.14). (B) Magnitude of absolute (unsigned) error for reproduction of 600 or 1400-msec intervals in the APTD or BAL sessions. APTD significantly (**) impaired the ability to accurately reproduce 1400-msec intervals. Error bars represent the SEM. (C) Mean response frequency as a function of mean reproduction time (200-msec time bins) of 600- or 1400-msec intervals in the APTD and BAL sessions. Data are shown separately for participants with low baseline availability of dopamine precursors (top) and high baseline availability of dopamine precursors (bottom). In the 1400-msec condition, opposing shifts (indicated by red arrow) were observed in the APTD session compared to the BAL session depending on participants' baseline precursor availability: APTD caused participants with low baseline dopamine availability to produce shorter reproduction times (i.e., to overestimate the elapse of time) but those with high baseline dopamine availability to produce longer times (i.e., to underestimate the elapse of time). Vertical dashed lines indicate the 600- and 1400-msec standard intervals.

times, $F(1,13)=0.004 ; p=.96 ;$ partial eta $=.001$, nor interaction between Drink and Interval Duration, $F(1,13)=$ $0.04 ; p=.84 ;$ partial eta $=.003$.

The repeated-measures ANOVA of absolute error revealed a significant main effect of Drink, $F(1,13)=10.01 ; p=.007$; partial eta $=.42$, indicating that the magnitude of error was far greater in the APTD session $(M=291.73 \mathrm{msec}, S E=$ 44.24) than the BAL session $(M=180.57 \mathrm{msec}, S E=$ 27.38). The analysis also revealed a main effect of Interval Duration, $F(1,13)=10.10 ; p=.007$; partial eta $=.42$, which was qualified by a Drink $\times$ Duration interaction, $F(1,13)=7.70 ; p=.015$; partial eta $=.36$. The interaction indicated that APTD significantly increased the magnitude of reproduction error in the $1400 \mathrm{msec}(p=.006)$, but not the $600 \mathrm{msec}(p=.397)$, condition (Figure $5 \mathrm{~B})$.

\section{APTD Effects on Temporal Reproduction as a Function of Baseline Dopamine Precursor Availability}

The significant effect of APTD on absolute error without a corresponding effect on mean reproduction time suggests that APTD caused some of the participants to underestimate interval duration and some of them to overestimate it, resulting in a net lack of change in reproduction time in the group as a whole. Because APTD has previously been shown to modulate temporal reproduction times in opposite directions depending upon participants' baseline level of dopamine precursor availability (Coull, Hwang, et al., 2013), we explored this hypothesis by dividing participants into low dopamine availability or high dopamine availability groups using a median split of the tyr: $\Sigma$ LNAA data. We then conducted a $2 \times 2 \times 2$ repeated-measures ANOVA on mean reproduction times, with Drink and Interval Duration as within-subject factors and Baseline Dopamine Availability as a between-subjects factor. This ANOVA confirmed the significant main effect of Interval Duration, $F(1,13)=192.89 ; p<.001 ;$ partial eta $=.94$, and the lack of a main effect of Drink, $F(1,13)=0.38$; $p=.55$; partial eta $=.03$. However, it did reveal a significant three-way interaction between Drink, Interval Duration, and Baseline Dopamine Availability, $F(1,13)=4.95 ; p<$ .05 ; partial eta $=.28$. This interaction revealed that, in the 
1400-msec condition, participants with low baseline dopamine availability had shorter reproduction times in the APTD session ( $M=1328.45 \mathrm{msec}, S E=148.6)$ compared to the BAL session $(M=1356.59 \mathrm{msec}, S E=70.62)$, whereas participants with high baseline dopamine availability had significantly longer reproduction times in the APTD session $(M=$ 1609.99 msec, $S E=180.2$ ) compared to the BAL session ( $M=1403.68 \mathrm{msec}, S E=100.4$; Figure 5C, right-hand $)$. There were no other significant main effects or interactions (all $p>.1$ ).

By contrast, analyses of $S D$ and absolute error showed that the pattern of results did not change whether baseline dopamine precursor availability was included in the ANOVA or not. For both $S D$ and absolute error, there was no significant main effect of Baseline Dopamine Precursor Availability, nor interaction with this factor (all $p>.1$ ).

This pattern of results suggests that the direction, although not magnitude, of reproduction error differed as a function of individual differences in underlying dopamine function. Nevertheless, the individual data points in Figure 4 indicate that baseline dopamine precursor availability values vary continuously rather than categorically. Therefore, splitting the participants into two discrete groups may be an underpowered way of analyzing the data because parametric fluctuations in baseline dopamine precursor levels across participants would be lost. We therefore reanalyzed mean reproduction times in SPSS using a linear mixed model, which allowed us to include the baseline level of dopamine precursor availability for each experimental session of our repeated-measures design as a continuous covariate. The model included drink (APTD/BAL) and interval duration (600/1400 msec) as fixed effects, the level of baseline dopamine precursor availability as a fixed covariate, and participant as a random effect. Drink and interval duration were modeled as repeated effects, and we used an unstructured covariance structure. First, by using chi-squared log likelihood ratio tests, we found that including baseline dopamine precursor availability in the model vastly improved the goodness of fit as compared to a model without this factor, $\chi^{2}(4)=$ $77.113, p<.0001$, justifying the inclusion of this factor. Akaike information criterion (AIC) values indicated that a model including the full factorial combination of all three factors (AIC $=707.62$ ) explained the data better than a model without the three-way interaction (AIC $=731.68)$, or without the two-way interactions (AIC $=779.98)$. This linear mixed model confirmed and reinforced the findings of the ANOVA. As well as the expected main effect of Duration, $F(1,26)=11.42, p<.05$, there was a significant interaction between Drink and Duration, $F(1,15)=5.62$, $p<.05$, which was qualified by a significant three-way interaction between Drink, Duration, and Baseline Dopamine Precursor Availability, $F(1,15)=6.93, p<.02$.

\section{Temporal Orienting}

The ANOVA of mean RTs in the temporal orienting task revealed significant main effects of Interval Duration, $F(1$, 16) $=74.46 ; p<.001 ;$ partial eta $=.82$, and Validity, $F(1$, 16) $=27.13 ; p<.001 ;$ partial eta $=.63$, that were qualified by an Interval Duration $\times$ Validity interaction, $F(1,16)=$ $38.71 ; p<.001$; partial eta $=.71$. Mean RTs were significantly slower in the invalid versus valid condition when targets were presented at $600 \mathrm{msec}$ but not $1400 \mathrm{msec}$ (Figure 6A), confirming many previous reports (e.g., Coull \& Nobre, 1998) that RTs are slowed more by invalid cueing when targets are presented prematurely at $600 \mathrm{msec}$ than when they are delayed at $1400 \mathrm{msec}$. Although there was also a significant main effect of the drink, $F(1,16)=$ $4.51 ; p=.05$; partial eta $=.22$, indicating that RTs were globally slower in the APTD session $(M=298.52 \mathrm{msec}$,

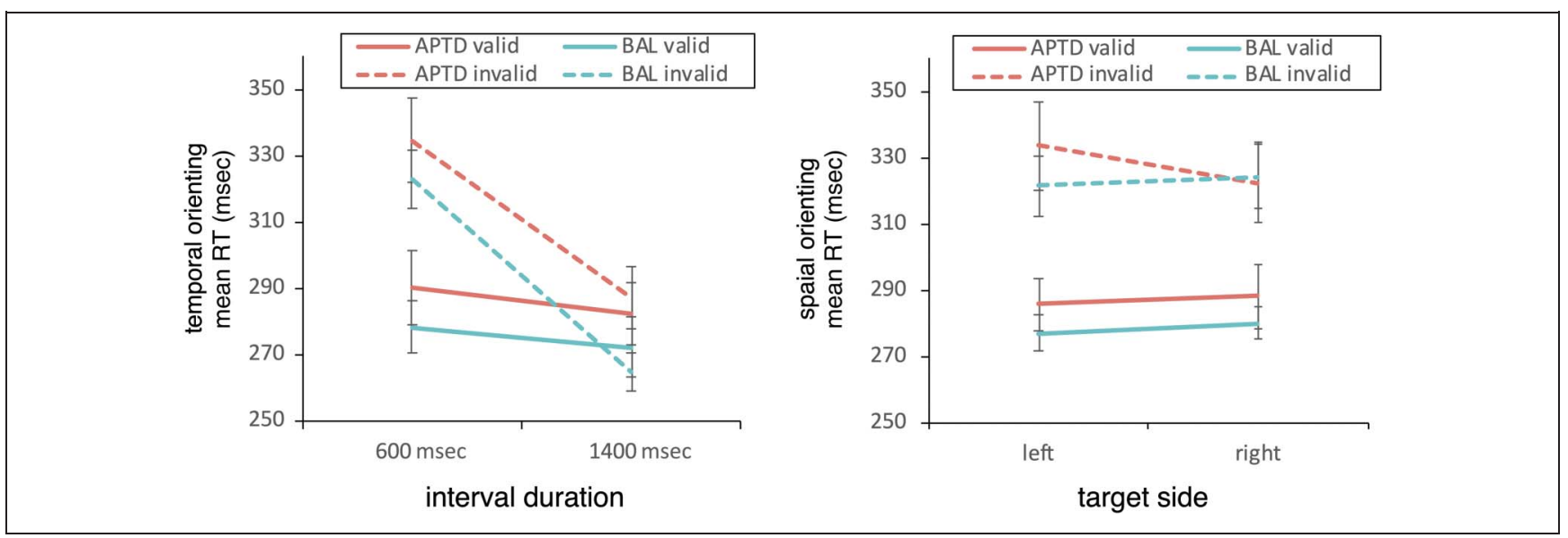

Figure 6. Orienting task. (A) Mean RTs in the temporal orienting task to targets appearing after an interval of 600 or 1400 msec. Target interval had been either correctly (valid) or incorrectly (invalid) predicted by a temporal cue. In both the APTD and BAL sessions, the RT cost of being invalidly cued was more pronounced when the target appeared unexpectedly early after only 600 msec, than when it appeared unexpectedly late after $1400 \mathrm{msec}$. Error bars represent the SEM. (B) Mean RTs in the spatial orienting task to targets appearing on either the left or right of the screen. Target position had been either correctly (valid) or incorrectly (invalid) predicted by a spatial cue. In both the APTD and BAL sessions, RTs were slower in invalid than valid trials. Error bars represent the SEM. 

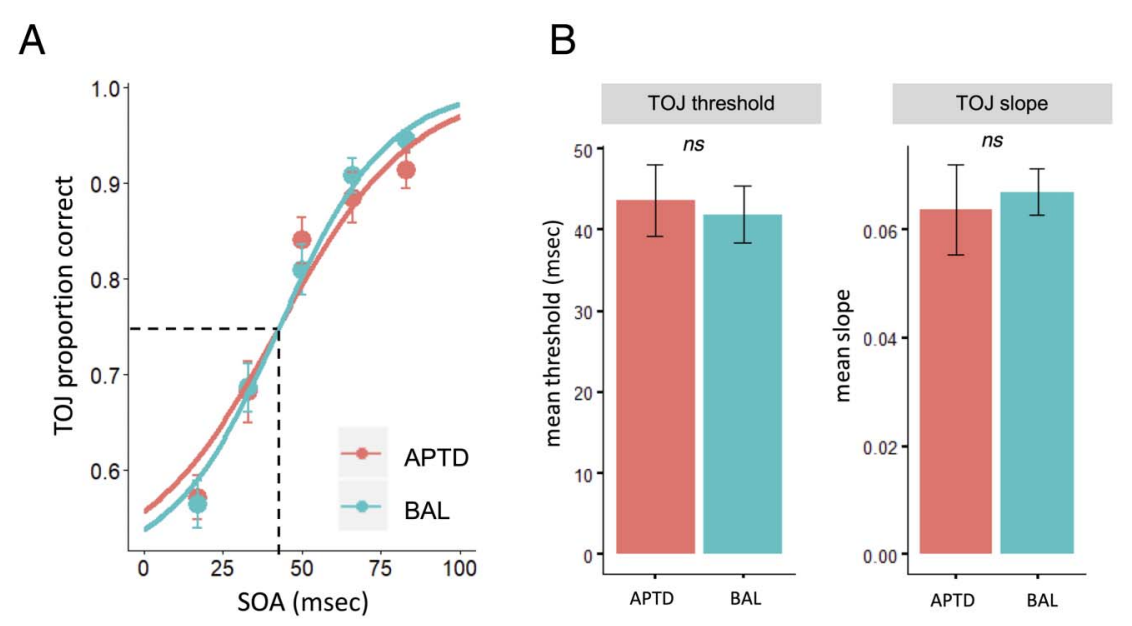

C

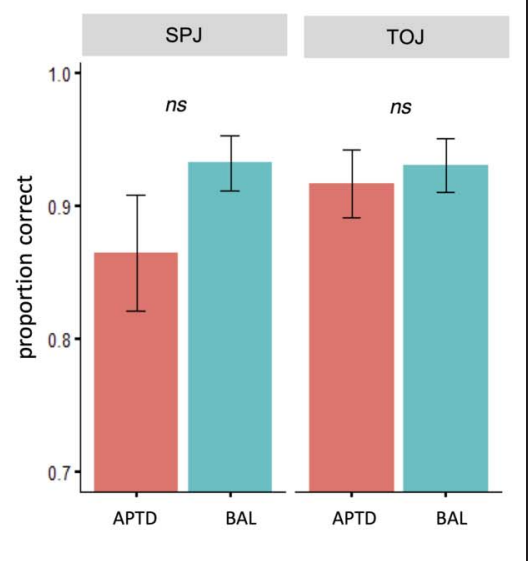

Figure 7. TOJ task. (A) Mean TOJ performance in the APTD and BAL sessions. Individual points indicate mean proportion correct at each of the SOAs, and curves represent the psychometric fit of these points. The temporal order threshold is defined as the SOA at which participants respond correctly $75 \%$ of the time (dashed lines) and represents the smallest SOA at which it is possible to accurately perceive temporal order. The slope of the psychometric curve indexes temporal sensitivity. Error bars represent the SEM. (B) Average temporal order threshold and average slope of individual psychometric curves in the APTD and BAL sessions. There were no significant (ns) differences between APTD and BAL sessions, in terms of either slope or threshold. Error bars represent the SEM. (C) Mean proportion of correct responses in the SPJ task and the performance-matched SOA condition of the TOJ task for the APTD and BAL sessions. There was ns effect of APTD on either the TOJ or SPJ tasks, nor differential effect as a function of task. Error bars represent the SEM.

$S E=9.44)$ than the BAL session $(M=284.49 \mathrm{msec}, S E=$ 6.72), drink did not significantly interact with any of the other factors (all $p>$.1). In other words, APTD did not modify the behavioral cost of having been invalidly cued to the wrong target interval. Neither the effects of the drink nor cue validity varied as a function of baseline dopamine precursor level (all $p>.1$ ), although, unexpectedly, the effects of interval duration did, $F(1,16)=7.11 ; p<.02$; partial eta $=.31$. The pattern of RTs revealed that the behavioral benefits of the hazard function for targets appearing after 1400 msec were greater in participants with low baseline dopamine availability $(M=312.09 \mathrm{msec}, S E=11.15$, and $M=272.82 \mathrm{msec}, S E=10.61$, for 600 - and 1400-msec intervals, respectively) than in participants with high availability $(M=300.92 \mathrm{msec}, S E=11.15$, and $M=280.19 \mathrm{msec}$, $S E=10.61$, for 600- and 1400-msec intervals respectively).

In the spatial orienting task, the ANOVA of mean RTs revealed a significant main effect of Validity, $F(1,16)=28.90$; $p<.001 ;$ partial eta $=.64$, indicating, as expected, that RTs were slower for invalid $(M=325.48 \mathrm{msec}, S E=9.65) \mathrm{com}$ pared to valid $(M=282.66 \mathrm{msec}, S E=6.05)$ trials (Figure 6B). There were no other significant main effects or interactions (all $p>1$ ), indicating that APTD did not modify the behavioral cost of having been invalidly cued to the wrong target side.

Analysis of mean RTs using linear mixed models confirmed that including baseline dopamine availability as a covariate in a full factorial model (with drink, interval duration, and validity as fixed effects and participant as a random effect) improved the goodness of fit compared to a similar model without this factor, both for temporal orienting, $\chi^{2}(8)=66.13, p<.0001$, and spatial orienting, $\chi^{2}(8)=59.15, p<.0001$. Importantly, however, there were no significant main effects of Drink, nor interaction between Drink and Baseline Dopamine Availability, for either temporal or spatial orienting (all $p>.1$ ).

\section{TOJ}

Inspection of individual participant's psychometric curves revealed a poor fit for two of the participants in the BAL session $\left(R^{2}=-.10\right.$ and .63$)$ who were therefore excluded from all subsequent TOJ statistical analyses. ${ }^{2}$ The average fit for the remaining participants in the BAL session was $0.91(S D=0.07)$ and in the APTD session was $0.81(S D=0.18)$.

The $2 \times 5 \times 2$ repeated-measures ANOVA conducted on the proportion of correct responses revealed, as expected, a main effect of SOA, $F(4,56)=113.78 ; p<.001$; partial eta $=.89$, indicating that the longer the SOA, the higher the proportion of correct responses (Figure 7A). No main effect of Drink was found, $F(1,14)=0.11 ; p=.75$; partial eta $=.01$, nor interaction between Drink and SOA, $F(4,56)=1.81 ; p=.14$; partial eta $=.12$, nor between Drink and Baseline Dopamine Availability, $F(1,14)=$ $0.20 ; p=.66$; partial eta $=.014$. All other main effects and interactions were similarly nonsignificant (all $p>.1$ ).

The $2 \times 2$ repeated-measures ANOVAs on the parameters derived from the psychometric curve fits (threshold and slope) confirmed these results (Figure $7 \mathrm{~B}$ ): There was no main effect of Drink on threshold, $F(1,14)=$ $0.40 ; p=.54$; partial eta $=.03$, or slope, $F(1,14)=0.15$; $p=.71$; partial eta $=.01$; nor of Baseline Dopamine Precursor Level on threshold, $F(1,14)=0.62 ; p=.44$; 
Table 1. Visual Analog Scale Mean (and $S D$ ) Values in the APTD or BAL Sessions at Baseline or after Cognitive Testing for Participants with Low or High Levels of Baseline Dopamine (DA) Precursor Availability

\begin{tabular}{|c|c|c|c|c|c|c|c|c|}
\hline \multirow[b]{2}{*}{$V A S$} & \multicolumn{2}{|c|}{ BAL Baseline } & \multicolumn{2}{|c|}{ BAL Test } & \multicolumn{2}{|c|}{ APTD Baseline } & \multicolumn{2}{|c|}{ APTD Test } \\
\hline & Low DA & High DA & Low DA & High DA & Low DA & High DA & Low DA & High DA \\
\hline Alert-drowsy & $0.3( \pm 0.15)$ & $0.33( \pm 0.27)$ & $0.49( \pm 0.25)$ & $0.45( \pm 0.24)$ & $0.2( \pm 0.19)$ & $0.35( \pm 0.22)$ & $0.5( \pm 0.22)$ & $0.55( \pm 0.3)$ \\
\hline Calm-excited & $0.22( \pm 0.13)$ & $0.3( \pm 0.23)$ & $0.27( \pm 0.16)$ & $0.22( \pm 0.2)$ & $0.29( \pm 0.2)$ & $0.32( \pm 0.21)$ & $0.18( \pm 0.11)$ & $0.31( \pm 0.3)$ \\
\hline $\begin{array}{l}\text { Contented- } \\
\text { discontented }\end{array}$ & $0.15( \pm 0.11)$ & $0.28( \pm 0.21)$ & $0.17( \pm 0.14)$ & $0.25( \pm 0.18)$ & $0.1( \pm 0.08)$ & $0.3( \pm 0.23)$ & $0.2( \pm 0.14)$ & $0.31( \pm 0.19)$ \\
\hline
\end{tabular}

partial eta $=.04$, or slope, $F(1,14)=0.04 ; p=.84 ;$ partial eta $=.003$. In addition, we found no interaction between the effects of the Drink and Baseline Dopamine Availability on threshold, $F(1,14)=1 ; p=.33$; partial eta $=.07$, or slope, $F(1,14)=1.08 ; p=.32$; partial eta $=.07$.

Analysis of TOJ parameters using linear mixed models confirmed that including baseline dopamine availability as a covariate in a model with drink as a fixed effect and participant as a random effect improved the goodness of fit compared to a similar model without this factor, both for threshold, $\chi^{2}(2)=23.73, p<.001$, and for slope, $\chi^{2}(2)=15.60, p<.001$. Importantly, however, there were no significant main effects of Drink, nor interaction between Drink and Baseline Dopamine Availability, for either threshold or slope (all $p>.1$ ).

In the SPJ condition, mean accuracy was high for all but one participant in the BAL session, who performed no better than chance (50\%) and so was excluded from SPJ analyses. To compare the effects of APTD on the processing of relative temporal versus spatial information, we compared performance in the SPJ task to the performance-matched SOA condition of the TOJ task (Figure 3C). Because the analysis was specifically designed to match performance across tasks, it was not surprising that, in the BAL session, there was no significant difference in the proportion of correct responses across tasks, $F(1,14)=1.60 ; p=.23$; partial eta $=.10$. However, there was also no significant effect of Drink, $F(1,14)=2.53 ; p=.13$; partial eta $=.15$, nor interaction between Drink and Task, $F(1,14)=1.77 ; p=.21$; partial eta $=.11$, indicating that APTD did not impair performance on either of these conditions (Figure $7 \mathrm{C}$ ).

\section{VAS}

There were no significant main effects of Drink, nor interactions between Drink and Dopamine precursor availability on any of the VAS values (Table 1 ). We found only a main effect of time for the alert-drowsy scale, $F(1,16)=16.38$; $p=.001$; partial eta $=.51$, which indicated that participants were more tired after cognitive testing than at baseline and a main effect of Dopamine precursor availability on the content-discontent scale, $F(1,16)=5.02 ; p=.04$; partial eta $=.24$, indicating that participants with high dopamine precursor availability were less content than participants with low baseline levels.

\section{DISCUSSION}

We compared, for the first time, the effects of dopaminergic manipulation on processing duration or temporal order in healthy volunteers. As compared to a nutritionally balanced drink, APTD produced a significant decrease in plasma concentrations of the dopamine precursor tyrosine compared to other neutral amino acids, leading to a reduction in brain availability of dopaminergic precursors. Importantly, the APTD drink had selective effects on discrete aspects of temporal perception: It impaired performance on a temporal reproduction task, but had no effect on TOJs or the ability to use temporal cues to speed RTs.

\section{APTD Differentially Affects Temporal Reproduction Times Depending on Baseline Dopamine Precursor Availability}

As compared to BAL, APTD significantly impaired the ability to accurately reproduce interval duration. Specifically, APTD increased the magnitude of absolute (unsigned) error during reproduction of 1400 -msec intervals. However, it had no corresponding effect on mean reproduction time. Therefore, although participants were less accurate in reproducing intervals, this error did not manifest itself as a consistent shift in a particular direction. Instead, some participants produced longer reproduction times after APTD, whereas others produced shorter times. By taking individual differences in baseline levels of dopamine precursor availability into account (see Coull, Hwang, et al., 2013), a clearer pattern emerged from the data: APTD shortened mean reproduction times of the 1400-msec interval in participants with low baseline dopamine precursor availability but lengthened them in participants with high availability. Put another way, APTD caused participants with low baseline availability to press the button sooner than they should have, meaning they had overestimated the amount of time that had elapsed. By contrast, for participants with high baseline availability, APTD caused them to press the button later than they should have, meaning they had underestimated the amount of time elapsed.

The effects of APTD particularly on 1400-msec reproduction times supports previous findings that APTD has significantly greater effects on longer duration stimuli 
(Coull, Hwang, et al., 2013). More importantly, the opposing effects of APTD in the two subgroups highlight the importance of accounting for individual differences in baseline or endogenous dopaminergic function in psychopharmacological studies, even within a relatively homogenous healthy population (see also Cools, 2019; Cools \& D'Esposito, 2011). In the sample as a whole, the opposing effects of APTD on mean reproduction times cancelled one another out and we might otherwise have concluded that APTD had no effect on mean reproduction time. However, by taking account of baseline dopamine precursor availability, we confirmed previous findings (Coull, Hwang, et al., 2013) that APTD affects duration processing as a function of underlying (endogenous) dopamine function.

Nevertheless, the pattern that emerged appeared, at first glance, to be the mirror image of results obtained in a prior APTD study of temporal reproduction, in which APTD lengthened reproduction times in participants with low baseline dopamine availability but shortened them in participants with high dopamine availability (Coull, Hwang, et al., 2013). Yet this apparent discrepancy can be easily reconciled by considering differences in the time at which the interval to be reproduced was initially encoded (i.e., learned and stored) in the two different paradigms. In the current study, the encoding of the interval to be reproduced took place at baseline, before dopamine precursor levels has been manipulated. The drink was therefore unable to affect the initial encoding of the standard interval, and any effects of APTD would have to reflect retrieval and reproduction of the learned interval. By contrast, the previous study used a roving standard, meaning APTD could have exerted its effects on encoding, as well as subsequent retrieval and reproduction (Coull, Hwang, et al., 2013). If so, shorter reproduction times could be the result of encoding a representation of duration into memory that was shorter than it should have been. In that study, APTD shortened reproduction times in participants with high baseline dopamine availability, suggesting these participants had underestimated the duration of the interval encoded into memory (Coull, Hwang, et al., 2013).

This compares to the results of the current study in which, as explained earlier, participants with high baseline dopamine precursor availability also underestimated duration after administration of APTD, which resulted in longer reproduction times. It seems, therefore, that APTD leads to underestimation of stimulus duration in participants with high baseline dopamine availability in both studies. However, depending on the design of the temporal reproduction task, this underestimation manifested itself at either the encoding or retrieval/reproduction phase of the task, producing opposite effects on reproduction times and thus reconciling the apparently paradoxical results. Finally, it is worth pointing out that, despite the fact that APTD could have affected both encoding and retrieval/reproduction in the previous study, which would have cancelled out any observable effect on reproduction time, significant effects were nevertheless obtained. This suggests that APTD might affect encoding of duration to a greater extent than retrieval/reproduction. Indeed, functional neuroimaging data confirm that APTD preferentially affects the encoding, rather than retrieval and discrimination, of the duration of visual stimuli (Coull et al., 2012).

\section{APTD Does Not Impair Temporal Order Processing}

This is the first study to examine the effects of a dopaminergic manipulation on temporal order processing in healthy volunteers. However, in contrast to APTD's effect on temporal reproduction, there were no significant effects of APTD on TOJ performance. Similarly, there was no effect of APTD on performance of an equally difficult spatial control task, in which participants had to judge the relative spatial position of two stimuli rather than their relative temporal order. These results indicate that decreases in dopaminergic function do not affect the ability to discriminate the relative location of two stimuli, whether these stimuli are separated in time or in space. Studies in patients with schizophrenia have previously revealed impairments in temporal order processing, which raises the possibility that dopamine could play a role in this process (Vatakis \& Bakou, 2015; Capa et al., 2014; Schwartz et al., 1991). However, the lack of effect of APTD on the TOJ task suggests that previously reported temporal order deficits in patients might be related to nondopaminergic aspects of the pathology. In support of this, Capa et al. (2014) found no correlation between schizophrenic patients' TOJ performance and dosage of antipsychotic medication, which suggests that TOJ impairments were independent of dopamine function. Of course, our findings do not completely rule out the possibility that dopamine might affect temporal order processing in healthy volunteers. Future experiments using more specific dopamine receptor antagonists could help reinforce or nuance our findings.

Another neurochemical candidate for mediating temporal order processing might be the noradrenergic system. Bellgrove et al. (2006) reported that temporal order processing deficits in ADHD were associated with the A2 allele of a Taq 1 polymorphism of the dopamine beta hydroxylase gene, which catalyses the conversion of dopamine to noradrenaline. The authors concluded that TOJ impairments in ADHD were caused by dysregulation of catecholamines in the brain, that is, dopamine and noradrenaline. Yet given the null effect of APTD on TOJ in our own study, it is possible that Bellgrove et al.'s (2006) genetic findings reflect dysfunction of the noradrenergic system rather than the dopaminergic one. Indeed, the noradrenergic system may prove a fruitful avenue for neurochemical investigation of temporal resolution and the succession of events more generally. For instance, difficult simultaneity judgments activate a region of brainstem consistent with the location of the locus coeruleus (Raizada \& Poldrack, 2008), the principal site of noradrenaline synthesis in the brain. The noradrenergic system has often been linked to 
attentional flexibility and reorienting (Sara, 2015; Sara \& Bouret, 2012; Aston-Jones, Rajkowski, \& Cohen, 1999), and a role in the segregation and ordering of events in time is consistent with this general framework. Indeed, psychopharmacological manipulation of the noradrenergic system in healthy volunteers with the $\alpha 2$ receptor agonist clonidine perturbs the ability to orient attention in time (Coull, Nobre, \& Frith, 2001), to detect an event if it occurs immediately (30 msec) after a previous one (Brown et al., 2016), or to detect events that occur in a specific order within a rapid visual presentation stream (Coull, Middleton, Robbins, \& Sahakian, 1995). Moreover, functional neuroimaging has revealed that clonidine affects performance of the latter task by modulating the functional connectivity between locus coereleus and inferior parietal cortex (Coull, Büchel, Friston, \& Frith, 1999). An interesting avenue for the future would be to measure the effects of noradrenergic manipulation on TOJ performance and to explore a possible dissociation in the effects of the noradrenergic and dopaminergic systems in temporal order processing versus duration processing, respectively.

\section{Dissociation between Dopaminergic Modulation of Duration versus Temporal Order Processing}

Our pattern of results shows that APTD impairs processing of duration, but not processing of temporal order. This suggests a neurochemical dissociation in the effects of dopamine on the perception of duration versus the perception of succession. An alternative explanation, however, is that APTD affected timing in the hundreds of milliseconds range (in the temporal reproduction task) but not in the tens of milliseconds range (in the TOJ task). In other words, the different effects of APTD on the two tasks could be because of differences in temporal scale rather than differences in processing metrical versus ordinal aspects of time. Nevertheless, Rammsayer (1993, 1997, 1999) has consistently shown that the dopaminergic antagonist haloperidol impairs duration estimates of intervals lasting only $50 \mathrm{msec}$, suggesting this explanation might not be sufficient. It should be remembered that our study is the first to compare the effects of dopaminergic manipulation on duration versus temporal order processing. Because of the pioneering nature of our study, we chose to use classic paradigms, each of which used the range of intervals that are typical for that paradigm. However, in order to conclusively compare the effects of APTD on the two types of timing, it would be preferable in the future to equate the interval range used across both paradigms, in other words, to compare TOJ performance to duration judgments of intervals that lasted only tens of milliseconds.

Given the well-established role of dopamine in motor control (Crocker, 1997; Kish, Shannak, \& Hornykiewicz, 1988; Marshall, Levitan, \& Stricker, 1976), we must also consider the possibility that our results simply reflect an effect of APTD on motor (temporal reproduction), rather than perceptual (TOJ), measures of timing. However,
APTD has previously been shown to impair performance on a perceptual task of duration discrimination (Coull et al., 2012). In addition, despite the fact that APTD globally slowed RTs in the temporal orienting task, it did not modulate the RT benefits of a temporal cue more specifically. This suggests that APTD does not have a general effect on any motor measure of timing. Participants benefitted as much from a valid temporal cue in the APTD session as in the BAL session (i.e., RTs were faster for valid vs. invalid cues), indicating that they were able to use the temporal cue to predict when the target would appear so as to speed RTs. By contrast, Tomassini et al. (2016) have shown that dopaminergic antagonists interfere with the ability to extract temporally predictable information from the environment in order to benefit RT performance. In particular, haloperidol impaired performance most when temporal uncertainty about the length of the variable cue-target interval was highest. It is possible that our temporal orienting paradigm, in which targets appear at one of two predicted intervals on the vast majority (80\%) of trials, might not provide sufficient uncertainty for the effects of APTD to be observed. Indeed, patients with Parkinson disease are able to use the temporally predictable information inherent in the elapse of time (the "hazard function") to speed oculomotor (Degos, Ameqrane, Rivaud-Péchoux, Pouget, \& Missal, 2018; de Hemptinne, Ivanoiu, Lefèvre, \& Missal, 2013; Jurkowski, Stepp, \& Hackley, 2005) or manual (Tomassini, Pollak, Edwards, \& Bestmann, 2019) responses to visual targets. However, as soon as the experimental context is manipulated to increase temporal uncertainty, then significant differences between patients and controls are unveiled (Tomassini et al., 2019). Alternatively, functional neuroimaging (Coull et al., 2012; Ellis et al., 2007) and radioligand binding studies (Leyton et al., 2004; Montgomery et al., 2003) indicate that APTD preferentially modulates activity in regions that are part of the motor corticostriatal loop (e.g., putamen and supplementary motor area) rather than the dorsolateral prefrontal loop (e.g., dorsolateral caudate and pFC). Therefore, the prefrontal circuits involved in the temporal reorienting of attention in temporally uncertain conditions (Coull, Cotti, \& Vidal, 2016; Coull et al., 2001) might be affected less by APTD than by antagonists of D1/D2 receptors such as haloperidol (Parker, Alberico, Miller, \& Narayanan, 2013). Future experiments examining the effects of, for example, haloperidol on temporal orienting in temporally certain versus uncertain experimental contexts would help resolve these questions.

It is tempting to suggest that if dopamine-related pathologies such as schizophrenia, Parkinson disease, or ADHD are associated with impairments in processing both duration and temporal order, then both forms of temporal processing might jointly depend upon the dopaminergic system. Although our temporal reproduction results confirm numerous findings that dopamine is involved in the perception of duration (Agostino \& Cheng, 2016; Soares et al., 2016; Coull et al., 2011; Meck, 1996), our TOJ results 
suggest this might not be the case for the perception of succession. Based on results of correlational and principal factor analyses, Rammsayer and Brandler $(2004,2007)$ had suggested that the ability to judge duration and temporal order are linked and may stem from a common timing mechanism (a so-called "master clock"). However, our APTD results support the more widely held view that duration and temporal order are functionally distinct processes and complement behavioral (Stetson, Fiesta, \& Eagleman, 2007), developmental (Levin, Israeli, \& Darom, 1978), and neuroanatomical (Schubotz \& von Cramon, 2001) findings by providing, for the first time, neurochemical evidence in healthy volunteers against the notion of a single master clock for processing both duration and succession.

\section{Acknowledgments}

All participants gave written informed consent, and the study was approved by the Comité de Protection des Personnes Nord-Ouest I at the University of Rouen (Protocol No. CPP 0013/2017-2016-A01598-43).

Reprint requests should be sent to Jennifer T. Coull, Laboratoire des Neurosciences Cognitives UMR 7291, Aix-Marseille University \& CNRS, 3 Place Victor Hugo, 13331 Marseille cedex 3, France, or via e-mail: jennifer.coull@univ-amu.fr.

\section{Author Contributions}

Morgane Chassignolle: Conceptualization; Data curation; Formal analysis; Investigation; Methodology; WritingOriginal draft. Ljubica Jovanovic: Data curation; Formal analysis; Investigation; Writing-Review \& editing. Catherine Schmidt-Mutter: Data curation; Investigation; Project administration; Resources. Guillaume Behr: Data curation; Investigation. Anne Giersch: Data curation; Funding acquisition; Investigation; Methodology; Project administration; Resources; Writing-Review \& editing. Jennifer T. Coull: Conceptualization; Data curation; Formal analysis; Funding acquisition; Investigation; Methodology; Project administration; Resources; Software; Supervision; Writing-Review \& editing.

\section{Funding Information}

Jennifer T. Coull, Agence Nationale de la Recherche (http:// dx.doi.org/10.13039/501100001665), grant number: ANR16-CE37-0004-02.

\section{Diversity in Citation Practices}

A retrospective analysis of the citations in every article published in this journal from 2010 to 2020 has revealed a persistent pattern of gender imbalance: Although the proportions of authorship teams (categorized by estimated gender identification of first author/last author) publishing in the Journal of Cognitive Neuroscience (JoCN) during this period were $\mathrm{M}(\mathrm{an}) / \mathrm{M}=.408, \mathrm{~W}($ oman $) / \mathrm{M}=.335$, $\mathrm{M} / \mathrm{W}=.108$, and $\mathrm{W} / \mathrm{W}=.149$, the comparable proportions for the articles that these authorship teams cited were $\mathrm{M} / \mathrm{M}=.579, \mathrm{~W} / \mathrm{M}=.243, \mathrm{M} / \mathrm{W}=0.102$, and $\mathrm{W} / \mathrm{W}=.076$ (Fulvio et al., JoCN, 33:1, pp. 3-7). Consequently, JoCN encourages all authors to consider gender balance explicitly when selecting which articles to cite and gives them the opportunity to report their article's gender citation balance. The authors of this article report its proportions of citations by gender category to be as follows: $\mathrm{M} / \mathrm{M}=.565$, $\mathrm{W} / \mathrm{M}=.274, \mathrm{M} / \mathrm{W}=.081$, and $\mathrm{W} / \mathrm{W}=.081$.

\section{Notes}

1. This correlation was maintained (and actually reinforced) in the entire sample of 22 participants for whom baseline blood samples were available for both experimental sessions (Spearman's $R=0.52, p=.007$ ). This consistent relationship suggests that individual differences in baseline dopamine precursor availability could be used as a useful proxy for endogenous dopamine function.

2. Please note that the pattern of results did not change even if the participant with a fit of $R^{2}=.63$ was included in the analyses.

\section{REFERENCES}

Agostino, P. V., \& Cheng, R.-K. (2016). Contributions of dopaminergic signaling to timing accuracy and precision. Current Opinion in Behavioral Sciences, 8, 153-160. DOI: https://doi.org/10.1016/j.cobeha.2016.02.013

Allman, M. J., \& Meck, W. H. (2012). Pathophysiological distortions in time perception and timed performance. Brain, 135, 656-677. DOI: https://doi.org/10.1093/brain/awr210, PMID: 21921020, PMCID: PMC3491636

Artieda, J., Pastor, M. A., Lacruz, F., \& Obeso, J. A. (1992). Temporal discrimination is abnormal in Parkinson's disease. Brain, 115, 199-210. DOI: https://doi.org/10.1093/brain/115 .1.199, PMID: 1559154

Aston-Jones, G., Rajkowski, J., \& Cohen, J. (1999). Role of locus coeruleus in attention and behavioral flexibility. Biological Psychiatry, 46, 1309-1320. DOI: https://doi.org/10.1016 /S0006-3223(99)00140-7, PMID: 10560036

Bellgrove, M. A., Mattingley, J. B., Hawi, Z., Mullins, C., Kirley, A., Gill, M., et al. (2006). Impaired temporal resolution of visual attention and dopamine beta hydroxylase genotype in attention-deficit/hyperactivity disorder. Biological Psychiatry, 60, 1039-1045. DOI: https://doi.org/10.1016/j.biopsych.2006 03.062, PMID: 16876143

Bolbecker, A. R., Westfall, D. R., Howell, J. M., Lackner, R. J., Carroll, C. A., O'Donnell, B. F., et al. (2014). Increased timing variability in schizophrenia and bipolar disorder. PLoS One, 9 , e97964. DOI: https://doi.org/10.1371/journal.pone.0097964, PMID: 24848559, PMCID: PMC 4029800

Bond, A., \& Lader, M. (1974). The use of analogue scales in rating subjective feelings. British Journal of Medical Psychology, 47, 211-218. DOI: https://doi.org/10.1111/j.2044-8341.1974 .tb02285.x

Breska, A., \& Ivry, R. B. (2018). Double dissociation of singleinterval and rhythmic temporal prediction in cerebellar degeneration and Parkinson's disease. Proceedings of the National Academy of Sciences, U.S.A., 115, 12283-12288. DOI: https://doi.org/10.1073/pnas.1810596115, PMID: 30425170, PMCID: PMC6275527 
Brown, S. B. R. E., Slagter, H. A., van Noorden, M. S., Giltay, E. J., van der Wee, N. J. A., \& Nieuwenhuis, S. (2016). Effects of clonidine and scopolamine on multiple target detection in rapid serial visual presentation. Psychopharmacology, 233, 341-350. DOI: https://doi.org/10.1007/s00213-015-4111-y, PMID: 26507194, PMCID: PMC 4700095

Capa, R. L., Duval, C. Z., Blaison, D., \& Giersch, A. (2014). Patients with schizophrenia selectively impaired in temporal order judgments. Schizophrenia Research, 156, 51-55. DOI: https://doi.org/10.1016/j.schres.2014.04.001, PMID: 24768441

Carroll, C. A., Boggs, J., O’Donnell, B. F., Shekhar, A., \& Hetrick, W. P. (2008). Temporal processing dysfunction in schizophrenia. Brain and Cognition, 67, 150-161. DOI: https://doi.org/10.1016/j.bandc.2007.12.005, PMID: 18262701, PMCID: PMC2512257

Ciullo, V., Piras, F., Vecchio, D., Banaj, N., Coull, J. T., \& Spalletta, G. (2018). Predictive timing disturbance is a precise marker of schizophrenia. Schizophrenia Research: Cognition, 12, 42-49. DOI: https://doi.org/10.1016/j.scog.2018.04.001, PMID: 29928596, PMCID: PMC6007042

Cools, R. (2019). Chemistry of the adaptive mind: Lessons from dopamine. Neuron, 104, 113-131. DOI: https://doi.org/10 .1016/j.neuron.2019.09.035, PMID: 31600509

Cools, R., \& D'Esposito, M. (2011). Inverted-U-shaped dopamine actions on human working memory and cognitive control. Biological Psychiatry, 69, e113-e125. DOI: https://doi.org /10.1016/j.biopsych.2011.03.028, PMID: 21531388, PMCID: PMC3111448

Coull, J. T., Büchel, C., Friston, K. J., \& Frith, C. D. (1999). Noradrenergically mediated plasticity in a human attentional neuronal network. Neuroimage, 10, 705-715. DOI: https:// doi.org/10.1006/nimg.1999.0513, PMID: 10600416

Coull, J. T., Cheng, R.-K., \& Meck, W. H. (2011). Neuroanatomical and neurochemical substrates of timing. Neuropsychopharmacology, 36, 3-25. DOI: https://doi.org/10.1038/npp.2010.113, PMID: 20668434, PMCID: PMC3055517

Coull, J. T., Cotti, J., \& Vidal, F. (2016). Differential roles for parietal and frontal cortices in fixed versus evolving temporal expectations: Dissociating prior from posterior temporal probabilities with fMRI. Neuroimage, 141, 40-51. DOI: https://doi.org/10.1016/j.neuroimage.2016.07.036, PMID: 27431757

Coull, J. T., Davranche, K., Nazarian, B., \& Vidal, F. (2013). Functional anatomy of timing differs for production versus prediction of time intervals. Neuropsychologia , 51, 309-319. DOI: https://doi.org/10.1016/j.neuropsychologia.2012.08.017, PMID: 22964490

Coull, J. T., Hwang, H. J., Leyton, M., \& Dagher, A. (2012). Dopamine precursor depletion impairs timing in healthy volunteers by attenuating activity in putamen and supplementary motor area. Journal of Neuroscience, 32, 16704-16715. DOI: https://doi.org/10.1523/JNEUROSCI.1258-12.2012, PMID: 23175824, PMCID: PMC6621775

Coull, J. T., Hwang, H. J., Leyton, M., \& Dagher, A. (2013). Dopaminergic modulation of motor timing in healthy volunteers differs as a function of baseline DA precursor availability. Timing \& Time Perception, 1, 77-98. DOI: https://doi.org/10.1163/22134468-00002005

Coull, J. T., Middleton, H. C., Robbins, T. W., \& Sahakian, B. J. (1995). Clonidine and diazepam have differential effects on tests of attention and learning. Psychopharmacology, 120, 322-332. DOI: https://doi.org/10.1007/BF02311180, PMID: 8524980

Coull, J. T., \& Nobre, A. C. (1998). Where and when to pay attention: The neural systems for directing attention to spatial locations and to time intervals as revealed by both PET and fMRI. Journal of Neuroscience, 18, 7426-7435. DOI:
https://doi.org/10.1523/JNEUROSCI.18-18-07426.1998, PMID: 9736662, PMCID: PMC6793260

Coull, J. T., Nobre, A. C., \& Frith, C. D. (2001). The noradrenergic $\alpha 2$ agonist clonidine modulates behavioural and neuroanatomical correlates of human attentional orienting and alerting. Cerebral Cortex, 11, 73-84. DOI: https://doi.org/10.1093/cercor/11.1.73, PMID: 11113036

Crocker, A. D. (1997). The regulation of motor control: An evaluation of the role of dopamine receptors in the substantia nigra. Reviews in the Neurosciences, 8 , 55-76. DOI: https://doi .org/10.1515/REVNEURO.1997.8.1.55, PMID: 9402645

Degos, B., Ameqrane, I., Rivaud-Péchoux, S., Pouget, P., \& Missal, M. (2018). Short-term temporal memory in idiopathic and Parkin-associated Parkinson's disease. Scientific Reports, 8, 7637. DOI: https://doi.org/10.1038/s41598-018-25751-8, PMID: 29769545, PMCID: PMC5956077

de Hemptinne, C., Ivanoiu, A., Lefèvre, P., \& Missal, M. (2013). How does Parkinson's disease and aging affect temporal expectation and the implicit timing of eye movements? Neuropsychologia, 51, 340-348. DOI: https://doi.org/10 .1016/j.neuropsychologia.2012.10.001, PMID: 23063965

Drew, M. R., Fairhurst, S., Malapani, C., Horvitz, J. C., \& Balsam, P. D. (2003). Effects of dopamine antagonists on the timing of two intervals. Pharmacology, Biochemistry, and Behavior, 75, 9-15. DOI: https://doi.org/10.1016/S0091-3057 (03)00036-4

Ellis, K. A., Mehta, M. A., Naga Venkatesha Murthy, P. J., McTavish, S. F. B., Nathan, P. J., \& Grasby, P. M. (2007). Tyrosine depletion alters cortical and limbic blood flow but does not modulate spatial working memory performance or task-related blood flow in humans. Human Brain Mapping, 28, 1136-1149. DOI: https://doi.org/10.1002/hbm.20339, PMID: 17290373, PMCID: PMC6871381

Elvevåg, B., McCormack, T., Gilbert, A., Brown, G. D. A., Weinberger, D. R., \& Goldberg, T. E. (2003). Duration judgements in patients with schizophrenia. Psychological Medicine, 33, 1249-1261. DOI: https://doi.org/10.1017/S0033291703008122, PMID: 14580079

Fraisse, P. (1984). Perception and estimation of time. Annual Review of Psychology, 35, 1-36. DOI: https://doi.org/10.1146 /annurev.ps.35.020184.000245, PMID: 6367623

Harmer, C. J., McTavish, S. F. B., Clark, L., Goodwin, G. M., \& Cowen, P. J. (2001). Tyrosine depletion attenuates dopamine function in healthy volunteers. Psychopharmacology, 154, 105-111. DOI: https://doi.org/10.1007/s002130000613, PMID: 11291999

Harrington, D. L., Haaland, K. Y., \& Hermanowitz, N. (1998). Temporal processing in the basal ganglia. Neuropsychology, 12, 3-12. DOI: https://doi.org/10.1037/0894-4105.12.1.3, PMID: 9460730

Jones, C. R. G., \& Jahanshahi, M. (2014). Contributions of the basal ganglia to temporal processing: Evidence from Parkinson's disease. Timing \& Time Perception, 2, 87-127. DOI: https://doi .org/10.1163/22134468-00002009

Jurkowski, A. J., Stepp, E., \& Hackley, S. A. (2005). Variable foreperiod deficits in Parkinson's disease: Dissociation across reflexive and voluntary behaviors. Brain and Cognition, 58, 49-61. DOI: https://doi.org/10.1016/j.bandc.2004.09.008, PMID: 15878726

Kanabus, M., Szelag, E., Rojek, E., \& Pöppel, E. (2002). Temporal order judgement for auditory and visual stimuli. Acta Neurobiologiae Experimentalis, 62, 263-270, PMID: 12659292

Kish, S. J., Shannak, K., \& Hornykiewicz, O. (1988). Uneven pattern of dopamine loss in the striatum of patients with idiopathic Parkinson's disease. New England Journal of Medicine, 318, 876-880. DOI: https://doi.org/10.1056 /NEJM198804073181402, PMID: 3352672 
Kvålseth, T. O. (1985). Cautionary note about $R^{2}$. American Statistician, 39, 279-285. DOI: https://doi.org/10.1080 /00031305.1985.10479448

Lake, J. I., \& Meck, W. H. (2013). Differential effects of amphetamine and haloperidol on temporal reproduction: Dopaminergic regulation of attention and clock speed. Neuropsychologia , 51, 284-292. DOI: https://doi.org/10 .1016/j.neuropsychologia.2012.09.014, PMID: 22982605

Lee, K.-H., Bhaker, R. S., Mysore, A., Parks, R. W., Birkett, P. B. L., \& Woodruff, P. W. R. (2009). Time perception and its neuropsychological correlates in patients with schizophrenia and in healthy volunteers. Psychiatry Research, 166, 174-183. DOI: https://doi.org/10.1016/j.psychres.2008.03.004, PMID: 19278734

Lee, M.-S., Kim, H.-S., \& Lyoo, C.-H. (2005). “Off” gait freezing and temporal discrimination threshold in patients with Parkinson disease. Neurology, 64, 670-674. DOI: https://doi .org/10.1212/01.WNL.0000151961.14861.BA, PMID: 15728290

Levi, D. M., Klein, S. A., \& Aitsebaomo, A. P. (1985). Vernier acuity, crowding and cortical magnification. Vision Research, 25, 963-977. DOI: https://doi.org/10.1016/0042-6989(85) 90207-X, PMID: 4049746

Levin, I., Israeli, E., \& Darom, E. (1978). The development of time concepts in young children: The relations between duration and succession. Child Development, 49, 755-764. DOI: https://doi.org/10.2307/1128245, PMID: 710191

Leyton, M. (2010). Acute phenylalanine/tyrosine depletion. In I. P. Stolerman (Ed.), Encyclopedia of psychopharmacology (pp. 1017-1021). Berlin, Heidelberg: Springer-Verlag. DOI: https://doi.org/10.1007/978-3-540-68706-1_33

Leyton, M., Dagher, A., Boileau, I., Casey, K., Baker, G. B., Diksic, M., et al. (2004). Decreasing amphetamine-induced dopamine release by acute phenylalanine/tyrosine depletion: A PET/[11C] raclopride study in healthy men. Neuropsychopharmacology, 29, 427-432. DOI: https://doi.org/10.1038/sj.npp.1300328, PMID: 14583741

Leyton, M., Young, S. N., Pihl, R. O., Etezadi, S., Lauze, C., Blier, P., et al. (2000). Effects on mood of acute phenylalanine/tyrosine depletion in healthy women. Neuropsychopharmacology, 22, 52-63. DOI: https://doi.org/10.1016/S0893-133X(99)00086-X, PMID: 10633491

MacDonald, C. J., \& Meck, W. H. (2005). Differential effects of clozapine and haloperidol on interval timing in the supraseconds range. Psychopharmacology, 182, 232-244. DOI: https://doi .org/10.1007/s00213-005-0074-8, PMID: 16001114

Malapani, C., Deweer, B., \& Gibbon, J. (2002). Separating storage from retrieval dysfunction of temporal memory in Parkinson's disease. Journal of Cognitive Neuroscience, 14, 311-322. DOI: https://doi.org/10.1162/089892902317236920, PMID: 11970794

Maricq, A. V., \& Church, R. M. (1983). The differential effects of haloperidol and methamphetamine on time estimation in the rat. Psychopharmacology, 79, 10-15. DOI: https://doi.org 10.1007/BF00433008, PMID: 6403957

Marshall, J. F., Levitan, D., \& Stricker, E. M. (1976). Activationinduced restoration of sensorimotor functions in rats with dopamine-depleting brain lesions. Journal of Comparative and Physiological Psychology, 90, 536-546. DOI: https://doi org/10.1037/h0077230, PMID: 8470

McTavish, S. F. B., Cowen, P. J., \& Sharp, T. (1999). Effect of a tyrosine-free amino acid mixture on regional brain catecholamine synthesis and release. Psychopharmacology, 141, 182-188. DOI: https://doi.org/10.1007/s002130050823, PMID: 9952043

Meck, W. H. (1986). Affinity for the dopamine D2 receptor predicts neuroleptic potency in decreasing the speed of an internal clock. Pharmacology, Biochemistry, and Behavior, 25, 1185-1189. DOI: https://doi.org/10.1016/0091-3057(86) 90109-7
Meck, W. H. (1996). Neuropharmacology of timing and time perception. Cognitive Brain Research, 3, 227-242. DOI: https:// doi.org/10.1016/0926-6410(96)00009-2, PMID: 8806025

Merchant, H., Luciana, M., Hooper, C., Majestic, S., \& Tuite, P. (2008). Interval timing and Parkinson's disease: Heterogeneity in temporal performance. Experimental Brain Research, 184, 233-248. DOI: https://doi.org/10.1007/s00221-007-1097-7, PMID: 17828600

Montgomery, A. J., McTavish, S. F. B., Cowen, P. J., \& Grasby, P. M. (2003). Reduction of brain dopamine concentration with dietary tyrosine plus phenylalanine depletion: An [11C] raclopride PET study. American Journal of Psychiatry, 160, 1887-1889. DOI: https://doi.org/10.1176/appi.ajp.160.10.1887, PMID: 14514507

Noreika, V., Falter, C. M., \& Rubia, K. (2013). Timing deficits in attention-deficit/hyperactivity disorder (ADHD): Evidence from neurocognitive and neuroimaging studies. Neuropsychologia, 51, 235-266. DOI: https://doi.org/10.1016/j.neuropsychologia .2012.09.036, PMID: 23022430

Oldendorf, W. H., \& Szabo, J. (1976). Amino acid assignment to one of three blood-brain barrier amino acid carriers. American Journal of Physiology, 230, 94-98. DOI: https://doi.org/10.1152 /ajplegacy.1976.230.1.94, PMID: 1251917

Parker, K. L., Alberico, S. L., Miller, A. D., \& Narayanan, N. S. (2013). Prefrontal D1 dopamine signaling is necessary for temporal expectation during reaction time performance. Neuroscience, 255, 246-254. DOI: https://doi.org/10.1016/j.neuroscience.2013 .09.057, PMID: 24120554, PMCID: PMC3856920

Pastor, M. A., Artieda, J., Jahanshahi, M., \& Obeso, J. A. (1992). Time estimation and reproduction is abnormal in Parkinson's disease. Brain, 115, 211-225. DOI: https://doi.org/10.1093 brain/115.1.211, PMID: 1559155

Pöppel, E. (1997). A hierarchical model of temporal perception. Trends in Cognitive Sciences, 1, 56-61. DOI: https://doi.org /10.1016/S1364-6613(97)01008-5, PMID: 21223864

Posner, M. I., Snyder, C. R., \& Davidson, B. J. (1980). Attention and the detection of signals. Journal of Experimental Psychology: General, 109, 160-174. DOI: https://doi.org /10.1037/0096-3445.109.2.160

Raizada, R. D. S., \& Poldrack, R. A. (2008). Challenge-driven attention: Interacting frontal and brainstem systems. Frontiers in Human Neuroscience, 1, 3. DOI: https://doi.org/10.3389 /neuro.09.003.2007, PMID: 18958217, PMCID: PMC2525983

Rammsayer, T. H. (1993). On dopaminergic modulation of temporal information processing. Biological Psychology, 36 209-222. DOI: https://doi.org/10.1016/0301-0511(93)90018-4, PMID: 8260566

Rammsayer, T. H. (1997). Are there dissociable roles of the mesostriatal and mesolimbocortical dopamine systems on temporal information processing in humans? Neuropsychobiology, 35, 36-45. DOI: https://doi.org /10.1159/000119328, PMID: 9018022

Rammsayer, T. H. (1999). Neuropharmacological evidence for different timing mechanisms in humans. Quarterly Journal of Experimental Psychology, 52, 273-286. DOI: https://doi .org/10.1080/713932708, PMID: 10467900

Rammsayer, T. H. (2009). Effects of pharmacologically induced dopamine-receptor stimulation on human temporal information processing. NeuroQuantology, 7, 103-113. DOI: https://doi.org/10.14704/nq.2009.7.1.212

Rammsayer, T. H., \& Brandler, S. (2004). Aspects of temporal information processing: A dimensional analysis. Psychological Research, 69, 115-123. DOI: https://doi.org/10.1007/s00426 -003-0164-3, PMID: 14758474

Rammsayer, T. H., \& Brandler, S. (2007). Performance on temporal information processing as an index of general intelligence. Intelligence, 35, 123-139. DOI: https://doi.org /10.1016/j.intell.2006.04.007 
Roy, M., Grondin, S., \& Roy, M.-A. (2012). Time perception disorders are related to working memory impairment in schizophrenia. Psychiatry Research, 200, 159-166. DOI: https://doi.org/10.1016/j.psychres.2012.06.008, PMID: 22862910

Sara, S. J. (2015). Locus coeruleus in time with the making of memories. Current Opinion in Neurobiology, 35, 87-94. DOI: https://doi.org/10.1016/j.conb.2015.07.004, PMID: 26241632

Sara, S. J., \& Bouret, S. (2012). Orienting and reorienting: The locus coeruleus mediates cognition through arousal. Neuron, 76, 130-141. DOI: https://doi.org/10.1016/j.neuron.2012 .09.011, PMID: 23040811

Schubotz, R. I., \& von Cramon, D. Y. (2001). Interval and ordinal properties of sequences are associated with distinct premotor areas. Cerebral Cortex, 11, 210-222. DOI: https://doi.org/10 .1093/cercor/11.3.210, PMID: 11230093

Schwartz, B. L., Deutsch, L. H., Cohen, C., Warden, D., \& Deutsch, S. I. (1991). Memory for temporal order in schizophrenia. Biological Psychiatry, 29, 329-339. DOI: https://doi.org/10 .1016/0006-3223(91)90218-B, PMID: 1674663

Smith, A., Taylor, E., Rogers, J. W., Newman, S., \& Rubia, K. (2002). Evidence for a pure time perception deficit in children with ADHD. Journal of Child Psychology and Psychiatry, 43, 529-542. DOI: https://doi.org/10.1111/1469-7610.00043, PMID: 12030598

Soares, S., Atallah, B. V., \& Paton, J. J. (2016). Midbrain dopamine neurons control judgment of time. Science, 354, 1273-1277. DOI: https://doi.org/10.1126/science.aah5234, PMID: 27940870

Stetson, C., Fiesta, M. P., \& Eagleman, D. M. (2007). Does time really slow down during a frightening event? PLoS One, 2, e1295. DOI: https://doi.org/10.1371/journal.pone.0001295, PMID: 18074019, PMCID: PMC2110887

Suarez, I., Lopera, F., Pineda, D., \& Casini, L. (2013). The cognitive structure of time estimation impairments in adults with attention deficit hyperactivity disorder. Cognitive Neuropsychology, 30, 195-207. DOI: https://doi.org/10 .1080/02643294.2013.842548, PMID: 24111987

Thoenes, S., \& Oberfeld, D. (2017). Meta-analysis of time perception and temporal processing in schizophrenia: Differential effects on precision and accuracy. Clinical Psychology Review, 54, 44-64. DOI: https://doi.org/10.1016 j.cpr.2017.03.007, PMID: 28391027

Tomassini, A., Pollak, T. A., Edwards, M. J., \& Bestmann, S. (2019). Learning from the past and expecting the future in Parkinsonism: Dopaminergic influence on predictions about the timing of future events. Neuropsychologia, 127, 9-18. DOI: https://doi.org/10.1016/j.neuropsychologia.2019.02.003, PMID: 30763591, PMCID: PMC6456720

Tomassini, A., Ruge, D., Galea, J. M., Penny, W., \& Bestmann, S. (2016). The role of dopamine in temporal uncertainty. Journal of Cognitive Neuroscience, 28, 96-110. DOI: https:// doi.org/10.1162/jocn a 00880, PMID: 26401816

Toplak, M. E., Rucklidge, J. J., Hetherington, R., John, S. C. F., \& Tannock, R. (2003). Time perception deficits in attentiondeficit/hyperactivity disorder and comorbid reading difficulties in child and adolescent samples. Journal of Child Psychology and Psychiatry, 44, 888-903. DOI: https://doi .org/10.1111/1469-7610.00173, PMID: 12959497

Torta, D. M. E., Castelli, L., Latini-Corazzini, L., Banche, A., Lopiano, L., \& Geminiani, G. (2010). Dissociation between time reproduction of actions and of intervals in patients with Parkinson's disease. Journal of Neurology, 257, 1356-1361. DOI: https://doi.org/10.1007/s00415-010-5532-5, PMID: 20352253

Ulrich, R., \& Miller, J. (2004). Threshold estimation in twoalternative forced-choice (2AFC) tasks: The Spearman-Kärber method. Perception \& Psychophysics, 66, 517-533. DOI: https://doi.org/10.3758/BF03194898, PMID: 15283075

Vatakis, A., \& Bakou, A. E. (2015). Distorted multisensory experiences of order and simultaneity. In A. Vatakis \& M. Allman (Eds.), Time distortions in mind: Temporal processing in clinical populations (pp. 1-36). Leiden: Brill. DOI: https://doi.org/10.1163/9789004230699_002

Wearden, J. H., \& Bray, S. (2001). Scalar timing without reference memory? Episodic temporal generalization and bisection in humans. Quarterly Journal of Experimental Psychology, 54, 289-309. DOI: https://doi.org/10.1080 /02724990042000173, PMID: 11764836

Westheimer, G., \& McKee, S. P. (1977). Spatial configurations for visual hyperacuity. Vision Research, 17, 941-947. DOI: https://doi.org/10.1016/0042-6989(77)90069-4, PMID: 595400

White, T. P., Wigton, R. L., Joyce, D. W., Bobin, T., Ferragamo, C., Wasim, N., et al. (2014). Eluding the illusion? Schizophrenia, dopamine and the McGurk effect. Frontiers in Human Neuroscience, 8,565 . DOI: https://doi.org/10.3389/fnhum 2014.00565, PMID: 25140138, PMCID: PMC4122162

Yang, B., Chan, R. C. K., Zou, X., Jing, J., Mai, J., \& Li, J. (2007). Time perception deficit in children with ADHD. Brain Research, 1170, 90-96. DOI: https://doi.org/10.1016/j.brainres 2007.07.021, PMID: 17669375 